\title{
Models and Analysis of Wire Explosions Using TRAC II Simulations
}

\author{
A. Pekker and D. B. Reisman
}

September 1, 2000

U.S. Department of Energy

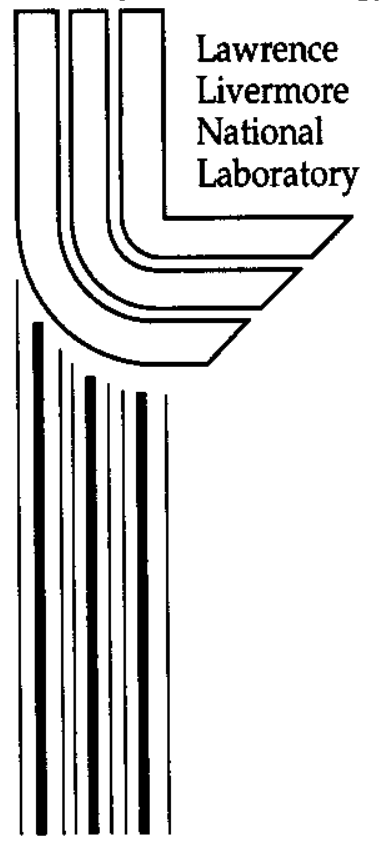




\section{DISCLAIMER}

This document was prepared as an account of work sponsored by an agency of the United States Government. Neither the United States Government nor the University of California nor any of their employees, makes any warranty, express or implied, or assumes any legal liability or responsibility for the accuracy, completeness, or usefulness of any information, apparatus, product, or process disclosed, or represents that its use would not infringe privately owned rights. Reference herein to any specific commercial product, process, or service by trade name, trademark, manufacturer, or otherwise, does not necessarily constitute or imply its endorsement, recommendation, or favoring by the United States Government or the University of California. The views and opinions of authors expressed herein do not necessarily state or reflect those of the United States Government or the University of California, and shall not be used for advertising or product endorsement purposes.

Work performed under the auspices of the U. S. Department of Energy by the University of California Lawrence Livermore National Laboratory under Contract W-7405-Eng-48.

This report has been reproduced

directly from the best available copy.

Available to DOE and DOE contractors from the

Office of Scientific and Technical Information

P.O. Box 62, Oak Ridge, TN 37831

Prices available from (423) 576-8401

http://apollo.osti.gov/bridge/

Available to the public from the

National Technical Information Service

U.S. Department of Commerce

5285 Port Royal Rd.,

Springfield, VA 22161

http://www.ntis.gov/

OR

Lawrence Livermore National Laboratory

Technical Information Department's Digital Library

http://www.llnl.gov/tid/Library.html 


\title{
Models and Analysis of Wire Explosions Using TRAC II Simulations
}

\author{
A. Pekker and D.B. Reisman \\ Lawrence Livermore National Laboratory \\ Livermore, California
}

September 1999 


\begin{abstract}
In order to understand the dynamics of Z-pinch imposions of thin wires in pulse-power accelerators, it is necessary to understand the physical process by which the initially solid wires are coverted into plasma by rising current. For this purpose, we model wire explosions using TRAC II, a two-demensional MHD code, in three distinct cases: pure tungsten, impure tungsten, and gold-plated tungsten. We compare our results - overall picture of the process, corona linear density, corona mass, and core expansion rate - to actual experiments performed at Sandia National Laboratory and Cornell University and present some explanations for the disagreements between our model and experimental observations.

In Chapter 1, we discuss model results for several current waveforms (consisting of a $5 \mathrm{kA} 50-150 \mathrm{~ns}$ pre-pulse and $80 \mathrm{kA} 80 \mathrm{~ns}$ main pulse) for a pure tungsten wire, showing that the initial temperature of the wire does not affect the dynamics of the explosion. This suggests that different experimental results for unheated and preheated tungsten wires are due to the expulsion of impurities in the preheated wire and not to a change in the material properties of tungsten. To match the experimental set-up more accurately,we model the explosion of a tungsten wire with impurties in Chapter 2. The overall process predicted by the model agrees with experiment, namely the shunting of the current through the impurities region before tungsten expansion begins; however, quantitative results disagree with experimental observations mostly because of the extreme shunting of the current through the impurities in our model. Finally, in Chapter 3, we compare the explosions in gold-plated tungsten, pure tungsten, and pure gold wires under high (100 kA in $60 \mathrm{~ns}$ ) and low ( $2 \mathrm{kA}$ in $270 \mathrm{~ns}$ ) currents, finding general agreement with experiment in the high-current case and a disagreement by a factor of ten in the lowcurrent case. In addition, due to the similar properties of the two metals, we find no vast differences among the three cases in the high-current case, while the single-metal wire expand faster and farther than the gold-plated wire in the low-current case. We believe that the disagreement between our model and experiment can be decreased by better modeling of tungsten impurities and by improvements in the conductivity and bonding models.
\end{abstract}




\section{Chapter 1}

\section{Tungsten Wires Without Impurities - Sandia Experiments}

\subsection{Introduction and Problem Set-Up}

In this chapter, we describe the Trac II modeling results for a single pure tungsten wire under four particular current shots. Actual experiments for this set-up have been performed at the Sandia National Laboratory. In Section 2, we discuss the coronal linear density, core expansion rate, and percent mass of the wire in the core at different times during wire explosions for these shots. In Section 3, we discuss the differences, or, rather, lack thereof, in these quantities for unheated and preheated wires.

We assume that the wires consist of pure tungsten, ignoring any impurities. This assumption is not entirely valid - SNL and Cornell University experimental data show that impurities that exist in the wires play an important role in the dynamics of wire explosions. These impurities are clearly manifested in experiments involving unheated and preheated wires - in the preheated wires, impurities are blown off during the pre-pulse, making the results, such as core expansion rate, different from those for unheated wires. In light of these differences, we have also simulated the explosions of tungsten wires with impurities: these results are discussed in detail in the next chapter.

The initial wire length is $2.0 \mathrm{~cm}$; the initial radius varies from $3.75 \mu \mathrm{m}$ 
to $3.835 \mu \mathrm{m}$. This difference in radius, amounting to less than $2.5 \%$, can be ignored in the analysis of our results. In three of the current shots, the wires are initially at room temperature (approximately $2.5 \times 10^{-2} \mathrm{eV}$ ); in the fourth shot, the wires are preheated to $1300 \mathrm{~K}$, or $0.112 \mathrm{eV}$.

The four current shots are shown in Figure 1.1. All current shots consist of a slow-rise pre-pulse, followed by a fast-rise main pulse, reaching up to 80 $\mathrm{kA}$. The slowest pre-pulse occurs in Shot 160 (3-4 kA in approximately 150 ns), followed by Shot 306 (6 kA in $70 \mathrm{~ns}$ ), and Shots 281 and 445 (3 kA in $50 \mathrm{~ns}$ ). Shots 281 and 445 have a very similar waveform and, therefore, are considered identical, except for the difference in the initial wire temperature between 281 and 445 .

In the actual experiment (but not in the model), the wires are held in a $2 \mathrm{~cm}$ radius circular array containing 240 wires. Therefore, the separation distance between the wires is approximately $2 \pi r / 240 \approx 520 \mu \mathrm{m}$. Our simulation is not applicable when the wire (core plus corona) radius exceeds the separation half-distance because at that point the individual wires become indistinguishable and form a joint plasma region in the wire array. Since for all four current shots, wire radius exceeds the separation half-distance of slightly more than $260 \mu \mathrm{m}$ at currents higher than $20 \mathrm{kA}$ (and, in all cases, repinching occurs before the peak current of $80 \mathrm{kA}$ is reached), we consider the dynamics of wire explosions only at $5,10,15$ and $20 \mathrm{kA}$.

We model the wire explosion using TRAC II, a magnetohydrodynamics (MHD) code, as a Lagrangian mesh consisting of a single, pure-tungsten region with 70 zones with a feathering factor of 0.95 . The code calculates quantities such as density, resistivity, and temperature at each mesh point. The results are written to a text file which is used by Tecplot 7.5, a graphing and analysis tool, to plot the results. A typical density versus radius plot appears in Figure 1.2. Each box corresponds to a mesh point; in the figure, the region between $r=0$ and $r=270 \mu \mathrm{m}$ (the first 46 boxes) corresponds to the dense core, while the region between $r=270$ and $r=790 \mu \mathrm{m}$ (the remaining 24 boxes) corresponds to the corona. Clearly, we can estimate that the corona begins at any one of the few mesh points near the core-corona boundary; this subjective choice introduces some error into our calculations. Finally, in order to measure the corona linear density $\rho_{L}$ between $r=R_{0}$ and $r=R_{1}$, we determine to which mesh points $R_{0}$ and $R_{1}$ correspond and use the Tecplot CFD Analyzer to numerically calculate $\rho_{L}=\int \rho(2 \pi r d r)$ between these mesh points. Tecplot CFD Analyzer produces very reasonable results; in our calculations, the error was always less than $10 \%$ compared to exact 
calculations. For instance, since wire mass is conserved during the explosion, numerical integration of the entire wire mass was within $10 \%$ of the original wire mass. In addition to linear density, we also consider the percent mass of the material in the corona, which is calculated simply by finding the linear density between the start and end of the corona and dividing it by the total linear density calculated using Tecplot.

Finally, we also calculate the expansion rate of the dense core during the pre-pulse. At various times during the pre-pulse, we estimated the end of the core region and the start of the plasma region and performed a linear regression on this distance versus time. In all cases, the correlation coefficient was above 0.99 . In addition, we estimated the expansion rate using the plots of the radii (mesh points) at various times, such as the one in Figure 1.3.

\subsection{Results for Unheated Wires}

During the current pre-pulse and main pulse, the tungsten wire continuously expands while at the same time the current is melting and vaporizing the material furthest away from the wire, thereby producing the plasma region. Figure 1.4 shows the density as a function of the radius (distance from the center of the wire) for Shot 281 when the current is 1,5 , and $20 \mathrm{kA}$; the curves are similar for the other two shots. As the figure demonstrates, most of the core expansion is due to the pre-pulse, i.e., up to the time when 5 $\mathrm{kA}$ current is reached, but expansion nevertheless continues up to the $20 \mathrm{kA}$ point. The small "kink" in the density right before the core-corona boundary is also observed in experiments.

For Shot 160 , the electron temperature in the core is less than $4 \mathrm{eV}$ and reaches up to $16 \mathrm{eV}$ in the corona. For shots 281 and 306, electron temperature in the core is less than $6 \mathrm{eV}$ and reaches up to $20 \mathrm{eV}$ in the corona. For all three shots, the radiation temperature is very similar to the electron temperature, never reaching above $20 \mathrm{eV}$ while the current is less than $20 \mathrm{kA}$.

We now consider the linear density of the corona as a function of the radius. This quantity is measured in Cornell experiments, and, for this reason, we choose to discuss it as well. In addition to the core expanding, the corona increases as well, both in volume and in mass. Linear density as a function of the radius for shots 160,281, and 306 is plotted in Figures 1.5, 1.6, and 1.7 , respectively. By "radius" we mean the radial distance from the start of 


\begin{tabular}{|l|l|l|l|}
\hline Current (kA) & $\begin{array}{c}\text { Shot 160 } \\
(\boldsymbol{\mu g} / \mathbf{c m})\end{array}$ & $\begin{array}{c}\text { Shot 160 } \\
(\boldsymbol{\mu g} / \mathbf{c m})\end{array}$ & $\begin{array}{c}\text { Shot 160 } \\
(\boldsymbol{\mu g} / \mathbf{c m})\end{array}$ \\
\hline 5 & 1.13051 & 0.80297 & 0.84143 \\
\hline 10 & 1.19370 & 0.94588 & 1.14581 \\
\hline 15 & 1.29027 & 1.02342 & 1.54221 \\
\hline 20 & 1.39307 & 1.11329 & 1.91438 \\
\hline
\end{tabular}

Table 1.1: Full corona linear density for Shots 160, 281, and 306.

\begin{tabular}{|l|l|l|l|}
\hline Current (kA) & Shot 160 (\%) & Shot 281 (\%) & Shot 306 (\%) \\
\hline 5 & 13.60 & 9.65 & 9.66 \\
\hline 10 & 14.37 & 11.36 & 13.18 \\
\hline 15 & 15.54 & 12.30 & 17.74 \\
\hline 20 & 16.76 & 13.38 & 22.01 \\
\hline
\end{tabular}

Table 1.2: Percent mass of the wire in the corona for Shots 160. 281, and 306.

the corona (not from the center of the wire).Since, for Shots 160 and 281, the initial density is $\rho=19.3 \mathrm{~g} / \mathrm{cm}^{3}$ and the initial radius $R=3.75 \mu \mathrm{m}$, the original linear density is $\rho_{L}=\rho\left(\pi R^{2}\right)=8.53 \mu \mathrm{g} / \mathrm{cm}$. For Shot 306, $\rho_{L}=8.92$ $\mu \mathrm{g} / \mathrm{cm}$.

For all current shots, a definite core is present at all times since the corona linear density is small compared to the total original linear density. Most of the corona is located close to the core; the increases in linear density become smaller and smaller as the radial distance from the start of the corona becomes greater and greater.

In Table 1.1, we present the calculated full corona linear density at different currents. Table 1.2 shows the percent mass of the wire in the corona, which is the ratio of the full corona linear density to the linear density of the whole (core plus corona) wire.

The data show that at currents up to $10 \mathrm{kA}$, the corona contains no more than $15 \%$ of the wire mass. The slowest rising pre-pulse, Shot 160. puts the most mass into the corona, followed by the medium pre-pulse, 306, and the fastest-rising pre-pulse, 281 . This suggests that at currents up to roughly $5-10 \mathrm{kA}$, the slowest-rising pre-pulse leads to the greatest melting of the core and the largest corona.

However, at 15 and $20 \mathrm{kA}$ currents, Shot 306 orertakes Shots 160 and 281 


\begin{tabular}{|l|l|l|}
\hline Shot 160 $(\mathrm{mm} / \mu \mathrm{s})$ & Shot 281 $(\mathrm{mm} / \mu \mathrm{s})$ & Shot 306 $(\mathrm{mm} / \mu \mathrm{s})$ \\
\hline $3.8-4.1$ & $3.4-3.7$ & $3.6-3.8$ \\
\hline
\end{tabular}

Table 1.3: Core radial expansion rates for Shots 160, 281, and 306.

and deposits significantly more mass into the corona than either of the other two. At $15 \mathrm{kA}$, Shot 306 corona contains $15 \%$ more mass than Shot 160 and $44 \%$ more than Shot 281 ; at $20 \mathrm{kA}$, Shot 306 corona contains $31 \%$ more mass than Shot 160 and $64 \%$ more mass than Shot 281.On average, the corona mass increases by $0.21-0.24 \%$ per Ampere of current (approximately $1.75-1.90 \%$ per ns) for Shots 160 and 281, but by $0.83 \%$ per Ampere (approximately $4.50 \%$ per ns) for Shot 306.

These dramatic differences may be due the shape of the current waveform between 5 and $20 \mathrm{kA}$, Figure 1.8. The rise time between 5 and $20 \mathrm{kA}$ is 23 and $18 \mathrm{~ns}$ for Shots 160 and 281, respectively, whereas it is more than $42 \mathrm{~ns}$ for Shot 306, i.e., roughly twice the time for the other two shots. Between 10 and $20 \mathrm{kA}$, the waveforms for Shots 160 and 281 are virtually identical, whereas the rise time for Shot 306 is still roughly twice that for the other two shots. In this interval, the mass in the corona increases by approximately $17 \%$ for both Shots 160 and 281, while it increases by $67 \%$ for Shot 306 . Therefore, we believe that at currents between $5-10 \mathrm{kA}$ and $20 \mathrm{kA}$, the heating time affects the amount of wire mass in the corona very significantly.

Table 1.3 shows the core radial expansion velocities; they range between 3.4 and $4.1 \mathrm{~mm} / \mu \mathrm{s}$. The data show that the slorrest-rising pulse, Shot 160, expands the fastest, followed by the medium-rise pulse, Shot 306, and finally, by Shot 281 . The explanation for this is that at low currents the magnetic pressure is small compared to electron pressure and, therefore, cannot constrain the plasma well (hence the fast expansion rate for Shot 160), whereas at higher currents, such as in Shots 281 and 306, it can constrain the plasma more effectively. These results are in reasonable agreement with Cornell experimental results. Cornell measurements show that tungsten expansion rates range between 1.9 and $3.8 \mathrm{~mm} / \mu \mathrm{s}$ and gold expansion rates range between 4.8 and $6.2 \mathrm{~mm} / \mu \mathrm{s}$. Impurities in tungsten wires used in experiments slow down the formation of the corona and hence produce a lower expansion rate than in pure tungsten wires which we consider in our models. In addition, since tungsten and gold have similar characteristics, and since gold wires tend to be much more pure than tungsten wires, the results seem to be in reasonable agreement. 


\begin{tabular}{|l|l|l|}
\hline Current (kA) & Shot 281 $(\boldsymbol{\mu g} / \mathbf{c m})$ & Shot 445 $(\boldsymbol{\mu g} / \mathbf{c m})$ \\
\hline 5 & 0.80297 & 0.80549 \\
\hline 10 & 0.94588 & 0.94869 \\
\hline 15 & 1.02342 & 1.02578 \\
\hline 20 & 1.11329 & 1.11398 \\
\hline
\end{tabular}

Table 1.4: Corona Linear Density for Shots 281 and 445.

\begin{tabular}{|l|l|l|}
\hline Current (kA) & Shot 281 (\%) & Shot 445 (\%) \\
\hline 5 & 9.65 & 9.67 \\
\hline 10 & 11.36 & 11.39 \\
\hline 15 & 12.30 & 12.31 \\
\hline 20 & 13.38 & 13.38 \\
\hline
\end{tabular}

Table 1.5: Percent Mass in the Corona for Shots 281 and 445.

\subsection{Results for Heated Wire}

We consider two current shots, 281 and 445 , which have very similar shapes, Figure 1.1. In both cases, the wire length is $2.0 \mathrm{~cm}$, the radius is $3.75 \mu \mathrm{m}$, and the composition is pure tungsten. The wire in Shot 281 is not preheated with a temperature of $2.5 \times 10^{-2} \mathrm{eV}$; the wire in Shot 445 is preheated to $1300 \mathrm{~K}$, or $0.112 \mathrm{eV}$. In the three characteristics we considered, corona linear density, percent mass in the corona, and core expansion rate, we found virtually no difference between the two shots.

Corona linear density for Shots 281 and 445 at the time when the current is 5, 10, 15, and $20 \mathrm{kA}$ is plotted in Figure 1.9; Tables 1.4 and 1.5 show the numerical results for full corona linear density and the percent mass in the corona, respectively. The data demonstrate that the differences between the two shots are less than $1 \%$. The core radial expansion rate for both shots is between 3.4 and $3.7 \mathrm{~mm} / \mu \mathrm{s}$.

The extreme similarity of the data for unheated and preheated wires demonstrates that preheating does not affect the dynamics of wire explosions for pure tungsten wires. These data disagree with experimental results, where preheated wires expand faster and have higher corona linear density than preheated wires. One possible explanation for these experimental results is that preheating to $1300 \mathrm{~K}$ increases the initial resistivity of tungsten by a factor of six and thus may affect the initial core heating rate. However. the 
model shows that this change in initial resistivity does not affect the initial core expansion rate, and, moreover, at the low currents throughout most of pre-pulse, this change should not play a significant role.

Instead, we believe that these experimental results are due to the fact that preheating also removes impurities present in the tungsten wires. By the time the wire is preheated to $1300 \mathrm{~K}$, SNL experiments show that among the impurities that are removed are $\mathrm{H}_{2}, \mathrm{H}_{2} \mathrm{O}, \mathrm{CO}$, and $\mathrm{CO}_{2}$. In unheated wires, these impurities form a skin layer and carry most of the current, thus reducing the amount of current penetrating the wire and inducing less ohmic heating and expansion as present in preheated or more pure wires. For this reason, we think, unheated wires expand slower and deposit less mass in the corona than preheated wires.

Since in this chapter we consider only pure tungsten wires, it makes sense that preheating does not affect the dynamics of wire explosions. In the next chapter we model wires with impurities by considering two regions, one consisting of unheated, pure tungsten, and the other consisting of heated carbon. 


\subsection{Figures for Chapter 1}

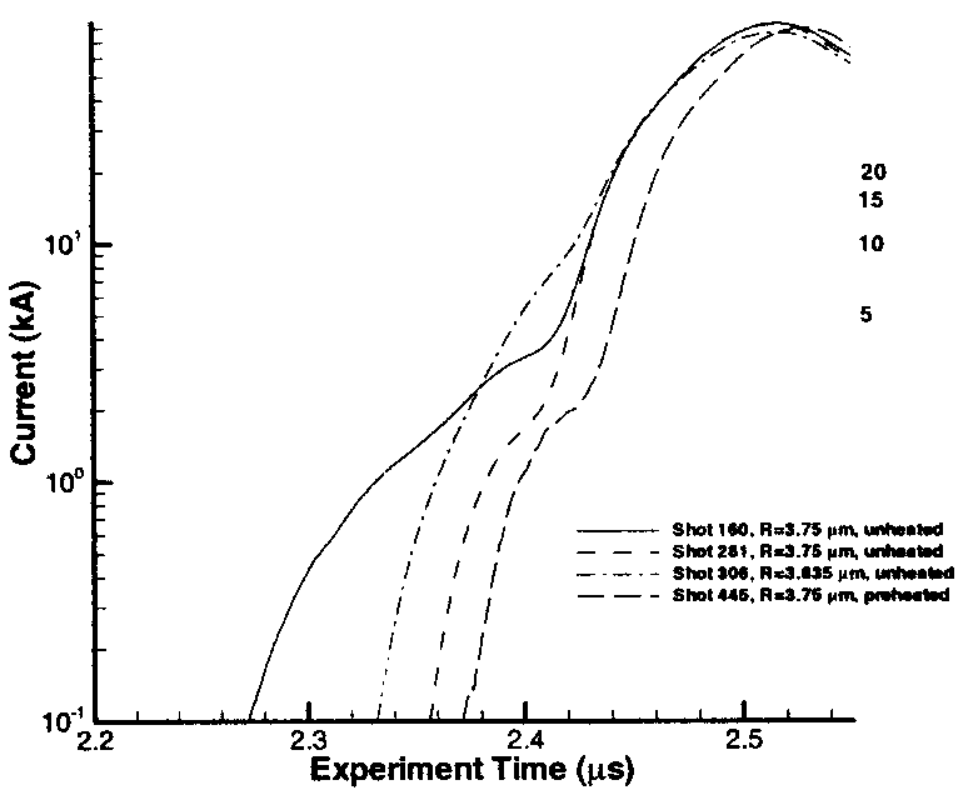

Figure 1.1: Four current shots considered. 


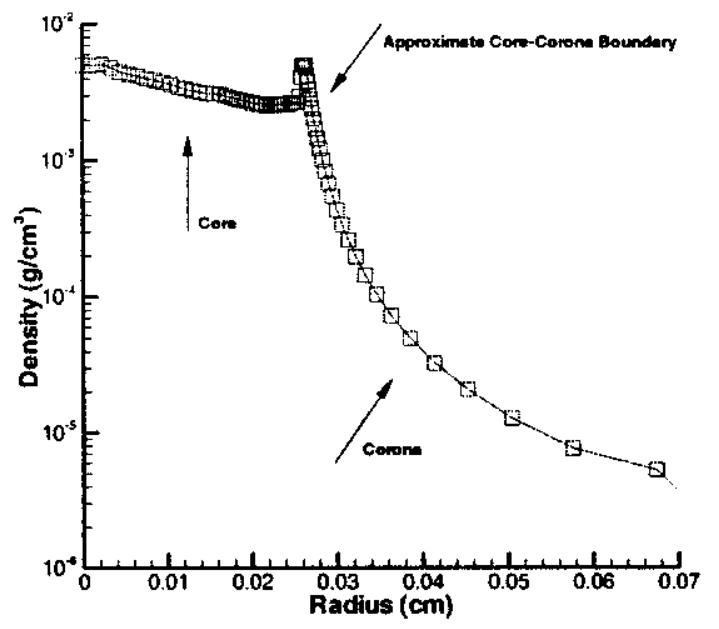

Figure 1.2: Density as a a function of the radius for Shot 281 at $I \approx 15 \mathrm{kA}$.

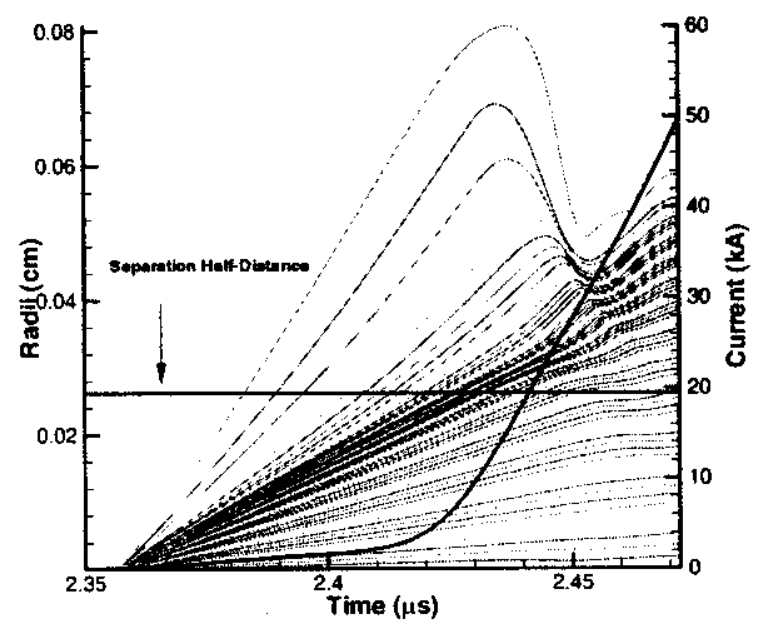

Figure 1.3: Radii (mesh points) as a function of time for Shot 281 . 


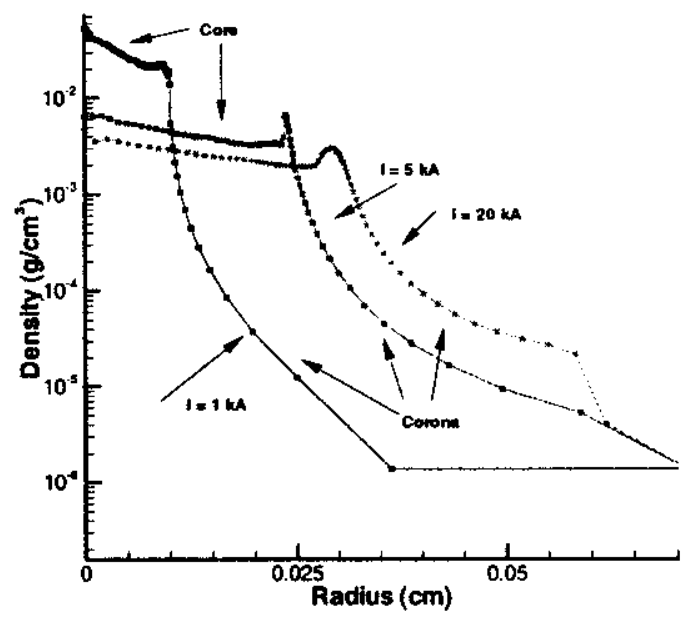

Figure 1.4: Density as a function of the radius at 1, 5, and $20 \mathrm{kA}$ for Shot 281.

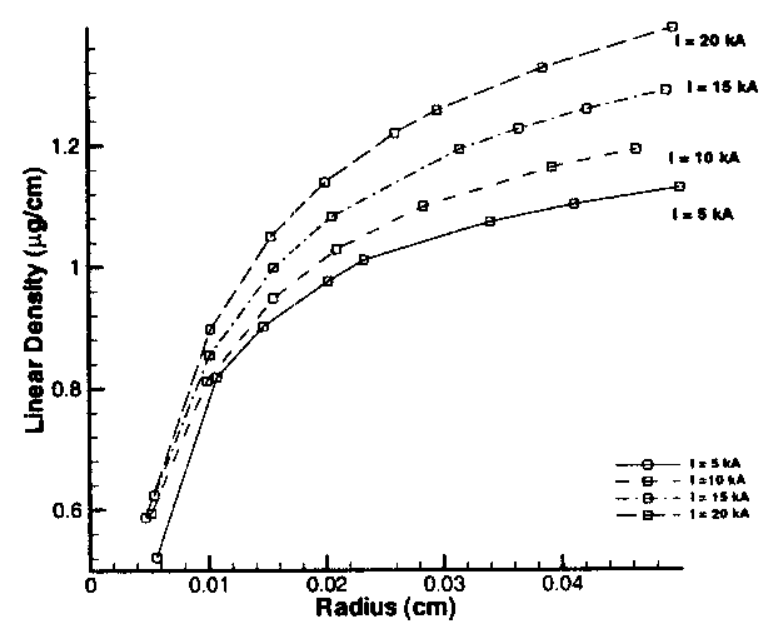

Figure 1.5: Linear density as a function of the radius from the start of the corona at 5, 10, 15, and $20 \mathrm{kA}$ for Shot 160. 


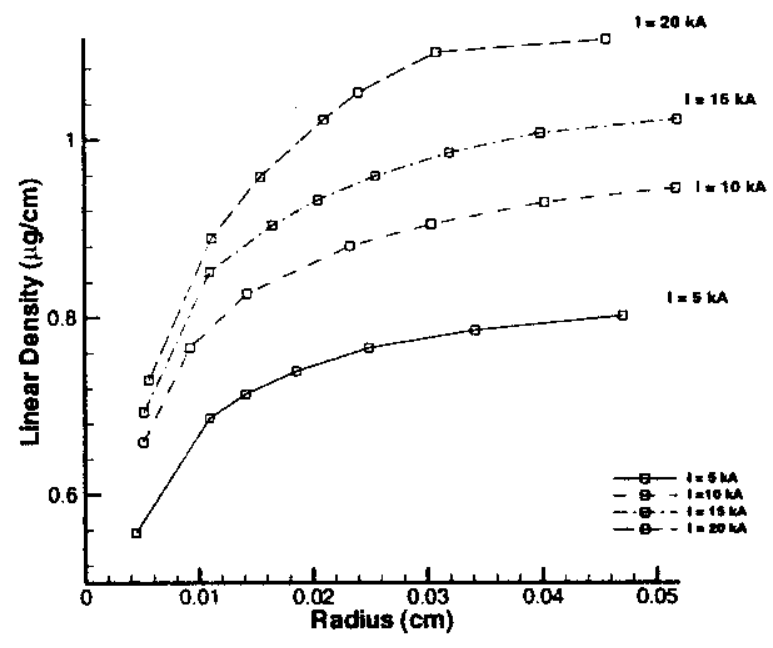

Figure 1.6: Linear density as a function of the radius from the start of the corona at 5, 10, 15, and $20 \mathrm{kA}$ for Shot 281.

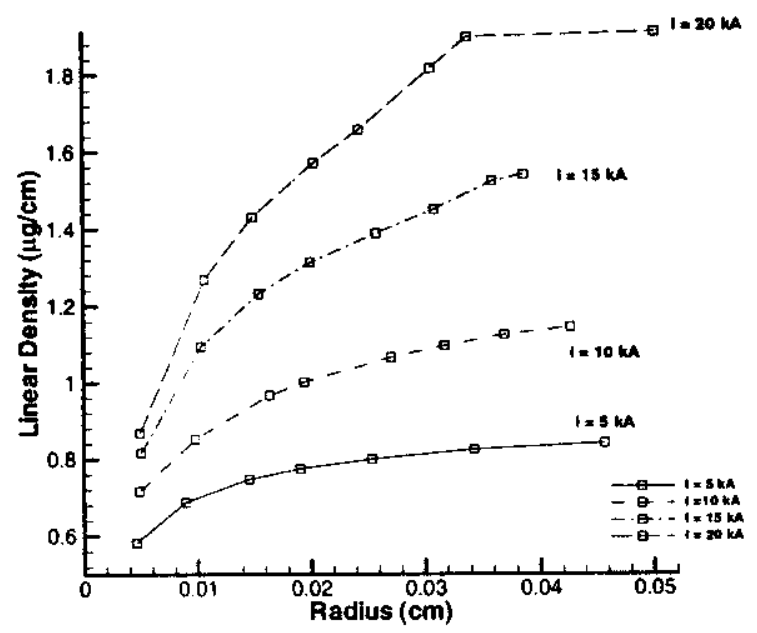

Figure 1.7: Linear density as a function of the radius from the start of the corona at 5, 10, 15, and $20 \mathrm{kA}$ for Shot 306 . 


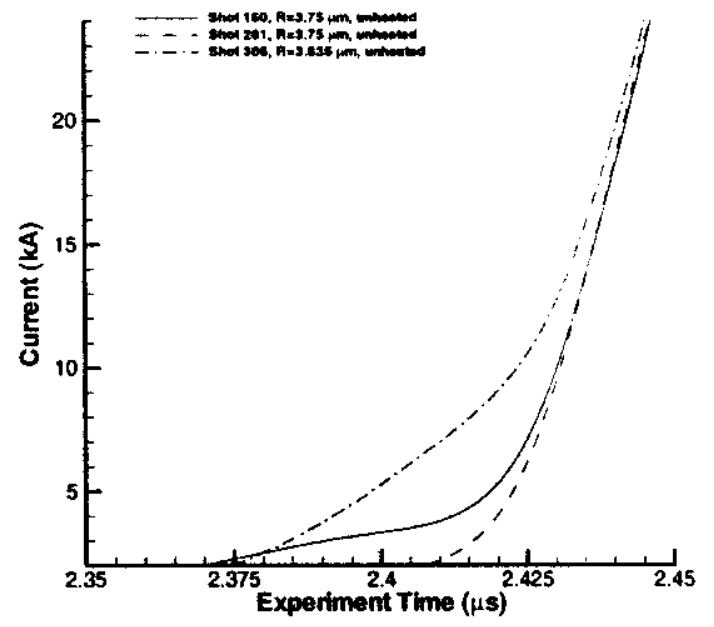

Figure 1.8: Shots 160,281 , and 306 between 5 and $20 \mathrm{kA}$.

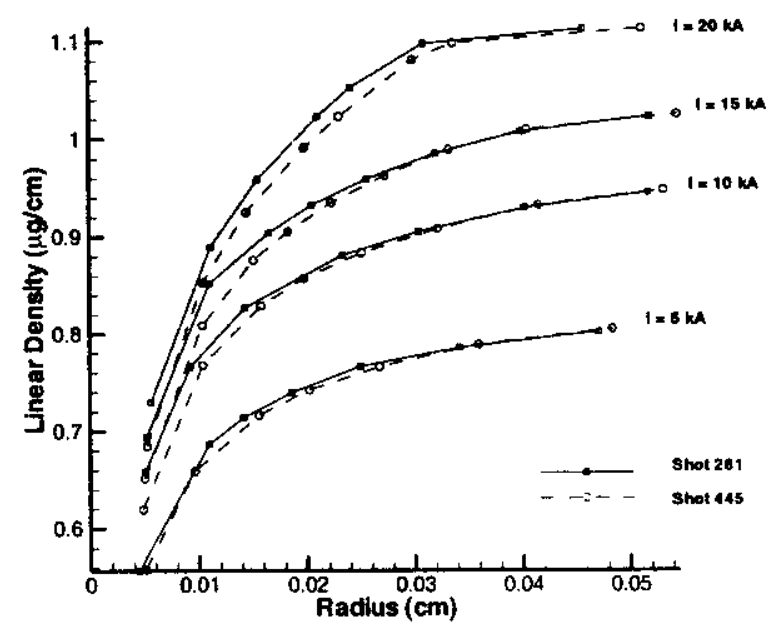

Figure 1.9: Corona linear density for Shots 281 (unheated) and 445 (preheated) at $5,10,15$, and $20 \mathrm{kA}$. 


\section{Chapter 2}

\section{Tungsten Wires With Impurities - Sandia Experiments}

\subsection{Introduction and Problem Set-Up}

As mentioned in the previous chapter, tungsten wires used in experiments contain impurities that significantly affect the rates of core expansion and corona growth. Thus, modeling explosions in pure tungsten wires presents only a first approximation to the actual process. In this chapter we discuss our model and its results for tungsten wires with impurities. Throughout this chapter, we concentrate on Shot 281 (50 ns pre-pulse to $3-4 \mathrm{kA}$ and $80 \mathrm{~ns}$ main pulse to $80 \mathrm{kA}$ ) with wire length of $2.0 \mathrm{~cm}$ and wire radius of $3.75 \mu \mathrm{m}$.

In Section 2.2, we model the wire as if it is composed of two radial regions. The inner "wire" region consists of pure tungsten at $T=2.5 \times 10^{-2} \mathrm{eV}$, $\rho=19.3 \mathrm{~g} / \mathrm{cm}$ and stretches between $r=0$ and $r=3.75 \mu \mathrm{m}$; the outer "impurities" region consists of pure carbon at $T=0.10 \mathrm{eV}, \rho=2.0 \times 10^{-5}$ $\mathrm{g} / \mathrm{cm}$ and stretches between $r=3.75$ and $r=53.75 \mu \mathrm{m}$. These parameters come from actual experiments performed at SNL. We use TRAC II as in the previous section with a Lagrangian mesh consisting of 70 zones and a feathering factor 0.95 for the tungsten region and 50 zones with a feathering factor of 0.90 for the carbon (or "impurities") region.

As shown in the experiments. the initial current flows through the carbon zones, which expand to a radius of $50-100 \mu \mathrm{m}$. Following the rapid expansion 
of the carbon zones, magnetic pressure repinches the carbon zones, and at 10 $\mathrm{kA}$, the entire wire, including tungsten begins to expand. Thus, the model appears to resemble the process observed in experiments.

In Section 2.3, following the suggestion of Dmitri Ryutov, we model the wire in the same way as in Section 2.2 with the added condition that when any carbon zone extends beyond the separation half-distance of $260 \mu \mathrm{m}$, we ignore the current flowing through this carbon zone, for, at this point, the impurities in the wire can combine with other impurities in the wire array and are effectively blown away from the wire. To ignore the current flowing through this carbon zone, we simply set the resistivity to an extremely large value such as $10^{12} \mathrm{~m} \Omega \cdot \mathrm{cm} / \mathrm{g}$.

While the overall expansion process is very similar under both models, the main difference in the process predicted by this adjusted model is that the tungsten region does not expand at all until a current of $20 \mathrm{kA}$ is reached; then, it expands very rapidly, converting virtually the entire core to plasma in $10 \mathrm{~ns}$.

In the figures and tables, we refer to the model of Section 2.2 as "I" and the model of Section 2.3 as "II."

\subsection{Results for Impure Wires I}

In this section, we use the two-material Lagrangian mesh where the impurities zones are not blown off if they extend beyond the separation half-distance.

Figure 2.1 shows the expansion of the $3.75 \mu \mathrm{m}$ tungsten wire covered by a $50 \mu \mathrm{m}$ carbon (impurities) region. The top fifty zones correspond to carbon impurities at $0.112 \mathrm{eV}$ and the remaining seventy zones correspond to pure, unheated tungsten. TRAC II output shows that $B \cdot r$, magnetic field times the radius, which, by Ampère's Law, is proportional to the current, is 3 - 4 orders of magnitude larger in the carbon zones than in the tungsten zones between the time the current is turned on until approximately $8 \mathrm{kA}$, Figure 2.2. Thus, the fact that the current flows mainly through the carbon region explains why there is no expansion in the tungsten wire but a rapid growth of the impurities region. However, around $8 \mathrm{kA}$, the magnetic pressure becomes large enough for the carbon zones to repinch. Intense ohmic heating is induced. melting

and vaporizing the tungsten and, thereby, producing plasma quickly. The core. however, does not expand at all. Maximum wire radius of $250 \mu \mathrm{m}$, 
$10 \mu \mathrm{m}$ less than the separation half-distance, is reached when the current is $34 \mathrm{kA}$.

The electron temperature in the carbon region stays below $60 \mathrm{eV}$ during most of the expansion, but just before the repinch it reaches almost 850 $\mathrm{eV}$. These temperatures are $1-2$ orders of magnitude higher than in pure tungsten wires in Chapter 1. During this pre-pulse, the tungsten heats up but still remains relatively cold, less than $0.5 \mathrm{eV}$. Following the repinch, however, the tungsten heats up very quickly, some zones increasing their temperature by two orders of magnitude in less than $10 \mathrm{~ns}$. The core is generally at $1-3 \mathrm{eV}$ and the corona is at $20 \mathrm{eV}$. At the same time, the carbon region maintains a broad and continuous range of temperatures; zones closest to the tungsten have temperatures near $45 \mathrm{eV}$ and those farthest from it, near $450 \mathrm{eV}$, again 1 - 1.5 orders magnitude higher than in pure tungsten wires. The exact results are shown in Figure 2.3. The radiation temperature stays well below the electron temperature during the expansion of the carbon region (less than 1 $\mathrm{eV}$ ) but is virtually equivalent to the electron temperature in both regions once the tungsten region begins to expand (i.e., after $8 \mathrm{kA}$ current is reached).

Figure 2.1 shows that the carbon zones extend beyond the separation half-distance during the pre-pulse which means that the impurities are blown away from the wire and, in reality, do not repinch. This is the reason that led us to consider the model with the carbon zones being blown off once they extend beyond the separation half-distance; the following section discusses this model.

As in the previous chapter, we calculated linear density as a function of the radius. However, in this model, the corona begins to form around 8 $\mathrm{kA}$ and reaches the maximum radius of $250 \mu \mathrm{m}$ - less than the separation half-distance - just before repinching at $34 \mathrm{kA}$. Thus, we consider the density when the current is $8,10,15,20,25,30$, and $34 \mathrm{kA}$. The plots of the usual density (from which we derive the linear density calculations) at several times are shown in Figure 2.4. The curve corresponding to $8 \mathrm{kA}$ shows that virtually all of the tungsten has not started melting, while the carbon zones are near their maximum radius - this is the situation immediately preceding the repinch of the carbon zones. The next three curves, corresponding to 10,20 , and $30 \mathrm{kA}$, show the expansion of the corona from its original radius of $40 \mu \mathrm{m}$ to $240 \mu \mathrm{m}$. At this time, however, the core remains fixed and the carbon zones do not expand at all. Finally, there is no appreciable difference between the times when the current is 30 and $34 \mathrm{kA}$, which corresponds to the maximum size of the corona. 


\begin{tabular}{|l|l|l|}
\hline Current (kA) & Linear Density $(\boldsymbol{\mu g} / \mathbf{c m})$ & Corona Mass (\%) \\
\hline 8 & $2.54267 \times 10^{-2}$ & 0.29 \\
\hline 10 & $2.61652 \times 10^{-1}$ & 3.10 \\
\hline 15 & 1.24969 & 14.70 \\
\hline 20 & 1.73892 & 21.23 \\
\hline 25 & 2.20563 & 27.07 \\
\hline 30 & 2.88953 & 35.03 \\
\hline 34 & 3.39043 & 40.68 \\
\hline
\end{tabular}

Table 2.1: Corona linear density and percent mass for Shot 281 with impurities I.

Since the full (core plus corona) wire radius is less than $45 \mu \mathrm{m}$ for currents less than $10 \mathrm{kA}$, Figure 2.5 shows the corona linear density as a function of the radius for $15,20,25,30$, and $34 \mathrm{kA}$. Linear density nearest to the core decreases as the corona expands, while the maximum linear density of almost $2.5-3.0 \mu \mathrm{g} / \mathrm{cm}$ is reached by the time the current is 30 and $34 \mathrm{kA}$. Table 2.1 shows the growth of the corona linear density and percent mass in the corona for the considered currents. Performing a linear regression with a correlation coefficient above 0.99 , we find that the current deposits $1.54 \%$ wire mass into the corona per $\mathrm{kA}$ of current (or, assuming the current is almost linear between 10 and $34 \mathrm{kA}, 1.43 \%$ per ns).

In summary, there are vast differences between the model results for tungsten wires with and without impurities, the main one being that most of the "action" (corona formation and core expansion) occurs in the pre-pulse in the pure-tungsten model, while the "action" occurs in the main pulse between 8 and $34 \mathrm{kA}$ in the impure-tungsten model. We believe that the model with impurities resembles the actual process more closely by showing the shunting of the current through the impurities region and its expansion beyond the separation half-distance. In the impure-tungsten model, the wire does not expand at all and begins to form a corona only after $8 \mathrm{kA}$ current is reached, while in the pure-tungsten model, the core begins to expand at 3.5 $-4.0 \mathrm{~mm} / \mu \mathrm{s}$ almost immediately and the corona begins forming soon after the current is turned on. The latter result, especially the expansion rates in Table 1.3, is comparable to experimental observations. Since the tungsten wire is heated for longer under the pure-tungsten model (all current goes through the wire at all times, unlike in the impurities model), the corona also expands by a factor of three further than in the impure-tungsten model, 
which reaches its peak radius of $250 \mu \mathrm{m}$. Nevertheless, since most of the heating and expansion in the pure-tungsten model goes on before $20 \mathrm{kA}$ current is reached, less than $15 \%$ of the wire mass is in the corona when the peak radius is reached, compared to more than $40 \%$ in the impure-tungsten model. As with Shot 306 in the pure-tungsten model, this results confirms that at higher currents solid wire is transformed into plasma much faster than at lower currents. Finally, linear density curves for pure tungsten wires, Figures $1.5-1.7$, resemble experimental results more closely than that for impure-tungsten, Figure 2.5. They show that most of the linear density is found near the core, and the growth in linear density decreases further away from the wire; in the impure-tungsten model, the opposite occurs: linear density growth increases further away from the core.

\subsection{Results for Impure Wires II}

In this section, we use the two-material Lagrangian mesh where the impurities zones are blown off if they extend beyond the separation half-distance. This model follows the observation from the previous section that the carbon zones extend well beyond the $260 \mu \mathrm{m}$ separation half-distance during their expansion; moreover, in the experiment, the carbon does not repinch although the model in the previous section predicts such a repinch. Thus, we simply set a very high resistivity, $10^{12} \mathrm{~m} \Omega \cdot \mathrm{cm} / \mathrm{g}$, when the carbon zone extends beyond the separation half-distance, effectively prohibiting current from flowing through it.

Figure 2.6 shows the radii as a function of time under this model. As in the pervious section, most of the current is flowing through the carbon zones during the pre-pulse as well as about $20 \mathrm{~ns}$ into the main pulse. Unlike in the previous section, however, there appears to be one tungsten zone that repinches before most of the plasma is formed. Plasma starts forming very quickly when the current reaches $22 \mathrm{kA}$; virtually all of the tungsten wire is turned into plasma by the time the current is $30 \mathrm{kA}$, i.e., in less than $10 \mathrm{~ns}$. The plasma expands until it reaches its maximum wire radius of $1.15 \mathrm{~mm}$ $(1150 \mu \mathrm{m})$ at $60 \mathrm{kA}$, i.e., less than $40 \mathrm{~ns}$ from the start of plasma formation.

The electron temperature of the carbon zones before the current reaches $22 \mathrm{kA}$ remains on the order of $10 \mathrm{eV}$ and always less than $40 \mathrm{eV}$ : the radiation temperature is eren smaller, staying below $10 \mathrm{eV}$. Howerer, once plasma begins forming, the electron temperature of some of the carbon zones exceeds 
$100 \mathrm{eV}$, but the radiation temperature remains less than $10 \mathrm{eV}$. As the carbon is blown off, tungsten gradually heats up, raising its electron and radiation temperatures to $0.5 \mathrm{eV}$ before they rise sharply to $100 \mathrm{eV}$ near $22 \mathrm{kA}$, as the plasma is forming. Less than $10 \mathrm{~ns}$ later, the temperature subside to $10-25 \mathrm{eV}$ for electrons and less than $10 \mathrm{eV}$ for radiation. Apparently this sharp rise in radiation temperature is responsible for the fast melting of the core and formation of the corona. The electron and radiation temperatures are shown in Figures 2.7 and 2.8. All the temperatures are comparable to those found in the model without blowing off the carbon zones, except the electron temperature of the carbon zones themselves. In the model in the previous section, the temperature of the carbon zones reaches $850 \mathrm{eV}$ before tungsten plasma forms and remains as high as $450 \mathrm{eV}$ after tungsten plasma is formed. This difference may be due to the fact that much more current is flowing through the carbon zones in the model without blowing off the carbon zones as opposed to the current model where there is virtually no current flowing through the carbon zones once they extend beyond the separation half-distance.

The different carbon zones' temperatures preceding the expansion of the tungsten - about $40 \mathrm{eV}$ in this model and about $400 \mathrm{eV}$ in the previous model - also explain why the tungsten in this model beings to expand at $22 \mathrm{kA}$, about $10-15 \mathrm{kA}$ "later" than in the model in the previous section. Once the tungsten begins to expand, plasma is produced very rapidly until the current reaches $30 \mathrm{kA}$, when the entire wire is converted to plasma. After that, the corona continues to expand until it reaches its maximum radius of $1.15 \mathrm{~mm}$ : in the process, the density becomes much more uniform as Figure 2.9 shows. In the following figure,we plot the linear density as a function of the radius for $30,40,50$ and $60 \mathrm{kA}$ current. The percent mass of wire in the corona is of course $100 \%$ as all of the tungsten is transformed into the corona. The main difference with regard to linear density between this and the previous model is that all of the wire is contained in the corona in this model, whereas only $40 \%$ is contained in the corona in the previous section.

In conclusion, although theoretically this model should approximate experiments closer than the pure-tungsten model considered in the previous chapter and the carbon-tungsten model without blow-off considered in the previous section, the results appear distinctly dissimilar from those obtained in the experiments. For instance, plasma formation occurs very quickly, such that there is virtually no core expansion and no definite core present. Experimentally, a dense core, containing most of the wire mass, is always present. 
Moreover, the wire radius extends beyond the separation half-distance much faster than in either of the two models, so the results are not directly applicable to the actual experiment. Overall, having examined three possible methods for two-dimensional simulations of tungsten wires with and without impurities, the process most closely resembling experiment is that of Section 2.3, while the one giving the most accurate results in comparison with experiment is that of the first chapter. 


\subsection{Figures for Chapter 2}

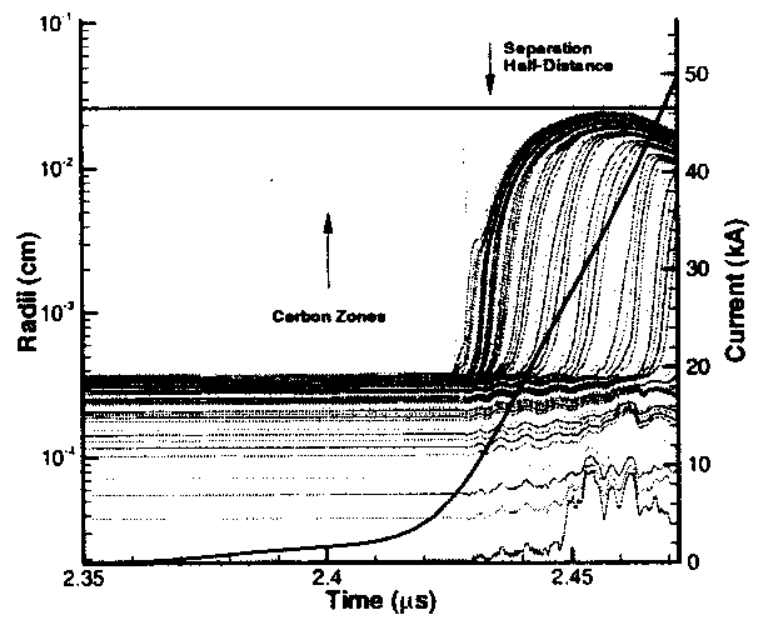

Figure 2.1: Radii (mesh points) as a function of time for Shot 281 with impurities I. 


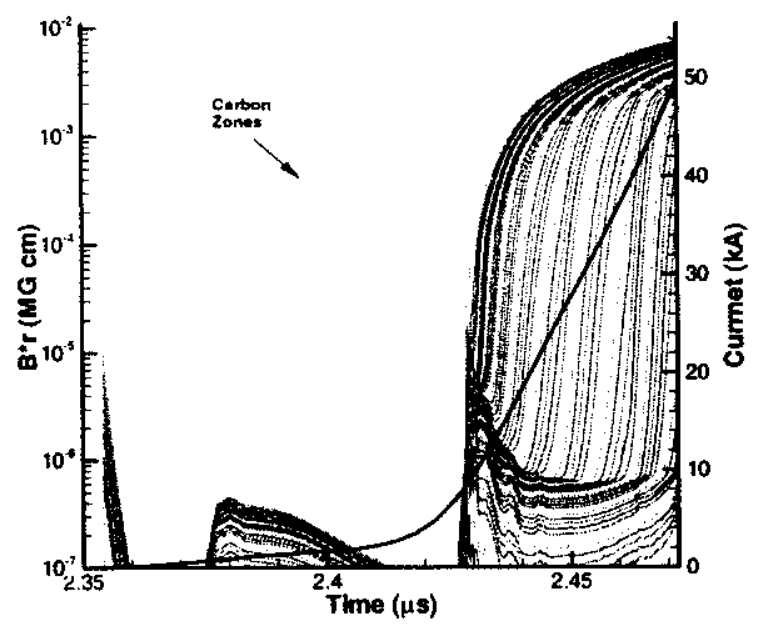

Figure 2.2: $B \cdot r$. magnetic field times the radius, as a function of time for Shot 281 with impurities I.

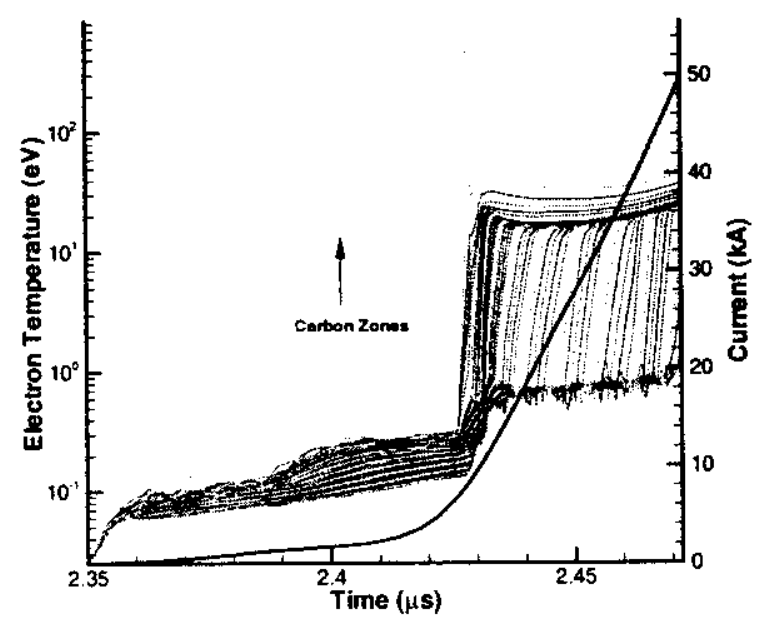

Figure 2.3: Electron temperature as a function of time for Shot 281 with impurities I. 


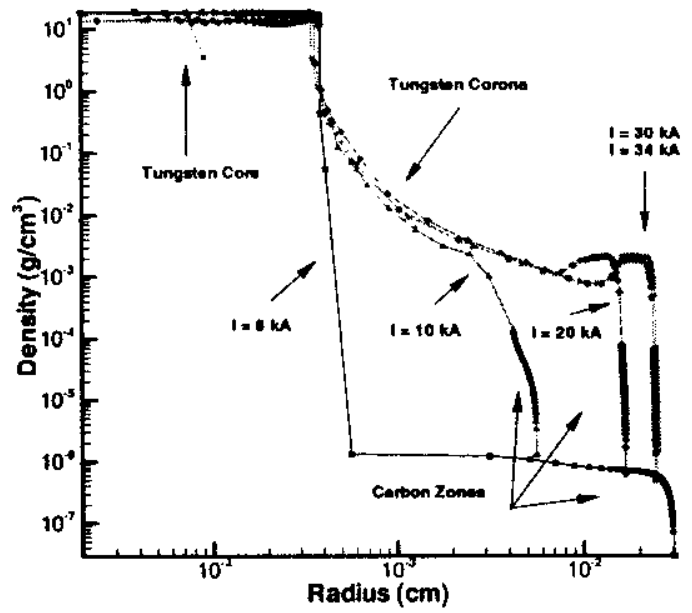

Figure 2.4: Density as a function of the radius at $8,10,20,30$, and $34 \mathrm{kA}$ for Shot 281 with impurities I.

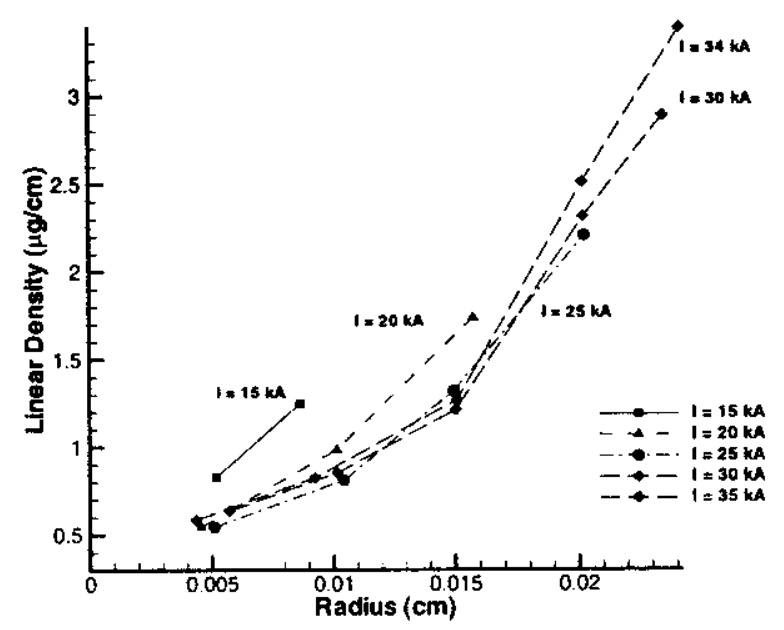

Figure 2.5: Corona linear density as a function of the radius (from the start of the corona) at $15,20,25,30$. and $34 \mathrm{kA}$ for Shot 281 with impurities I. 


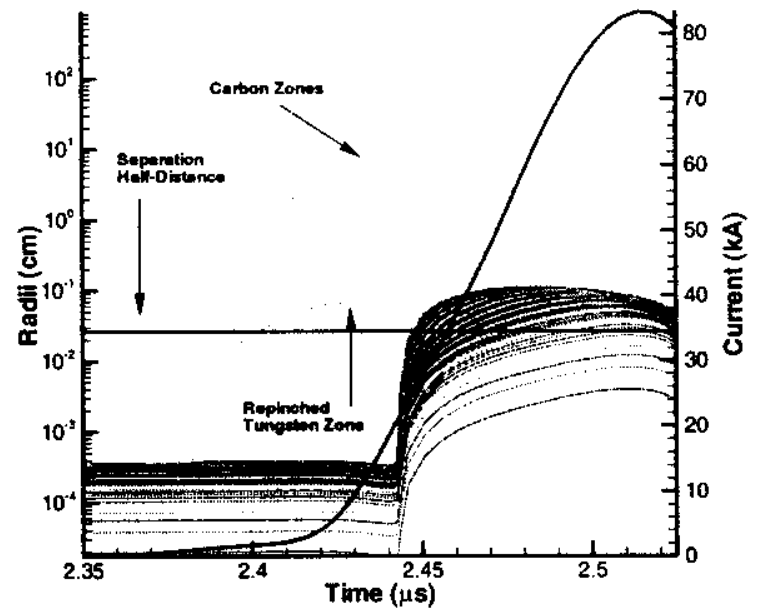

Figure 2.6: Radii (mesh points) as a function of time for Shot 281 with impurities II.

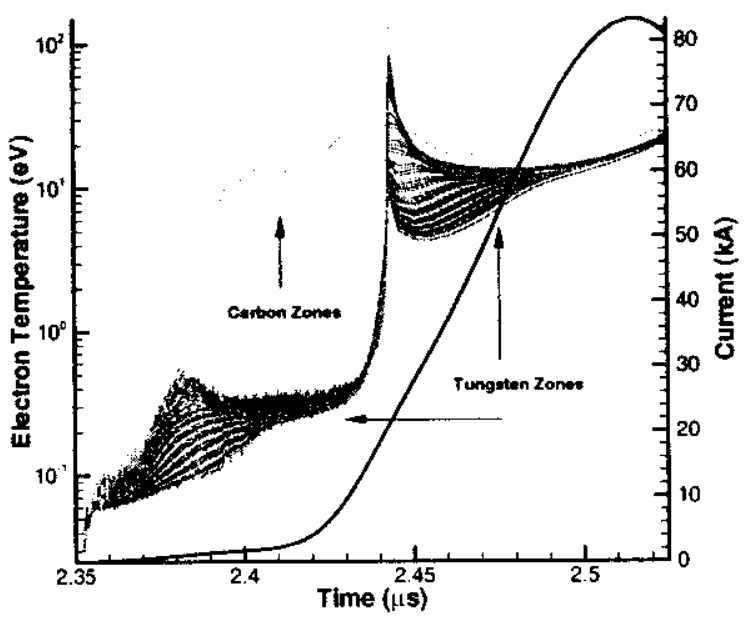

Figure 2.7: Electron temperature as a function of time for Shot 281 with impurities II. 


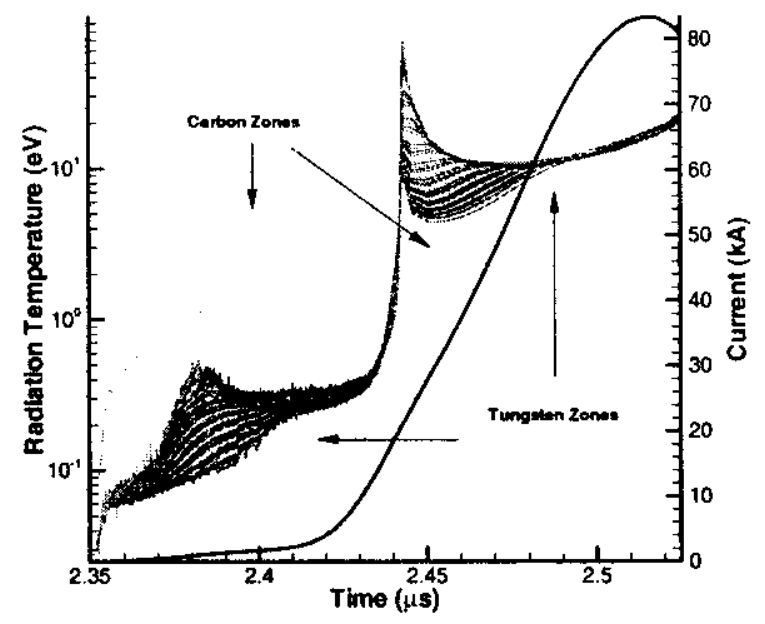

Figure 2.8: Radiation temperature as a function of time for Shot 281 with impurities II.

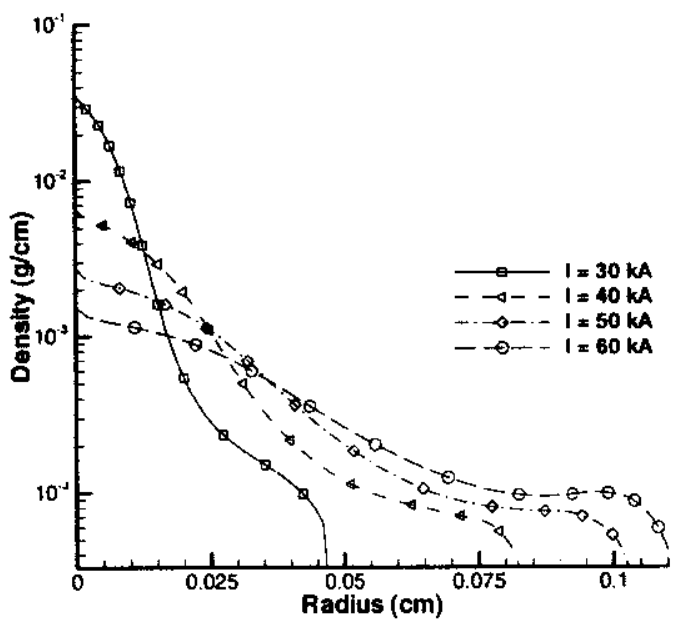

Figure 2.9: Density as a function of the radius at $30,40,50$, and $60 \mathrm{kA}$ for Shot 281 with impurities II. 


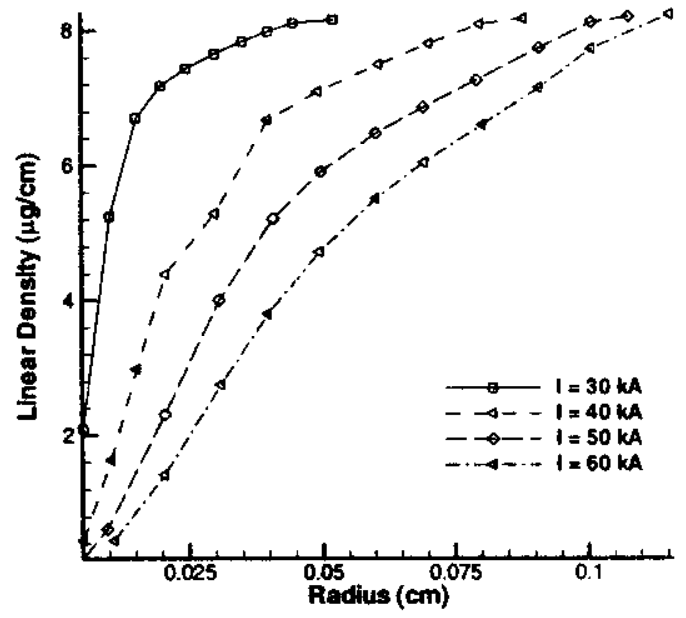

Figure 2.10: Linear density as a function of the radius at 30, 40, 50 and 60 $\mathrm{kA}$ for Shot 281 with impurities II. 


\section{Chapter 3}

\section{Gold-Plated, Pure Tungsten and Pure Gold Wires}

\subsection{Introduction and Problem Set-Up}

In this chapter we consider $10.247 \mu \mathrm{m}$-radius $5 \%$ gold-plated tungsten, pure tungsten, and pure gold wires. We choose to model these three cases because they should provide a good comparison and because there is experimental data from Cornell University for the $5 \%$ gold-plated tungsten wires.

As before, the wire length is $2.0 \mathrm{~cm}$ and the wires are unheated at the temperature of $2.5 \times 10^{-2} \mathrm{eV}$. We consider two current shots: the linear "high-current" Shot 1, which reaches $100 \mathrm{kA}$ in $60 \mathrm{~ns}$, and the linear "lowcurrent" Shot 2, which reaches $2 \mathrm{kA}$ in $267.6 \mathrm{~ns}$. For pure wires, we use TRAC II with a Lagrangian 120-zone mesh. For the gold-plated wires, we use TRAC II with the two-region Lagrangian mesh, 70 zones in the tungsten region and 50 zones in the gold region. Since both gold and tungsten have the same density of $19.3 \mathrm{~g} / \mathrm{cm}^{3}$, the initial linear density is $6.37 \times 10^{-5} \mathrm{~g} / \mathrm{cm}$. In the gold-plated case, the tungsten region is between 0 and $10 \mu \mathrm{m}$ and the gold region is between 10 and $10.247 \mu \mathrm{m}$, corresponding to $5 \%$ of the mass as desired.

In Cornell experiments, the "high-current" experiment is performed for a single wire while the "low-current" experiment is performed for two wires (namely, the total current at $267.6 \mathrm{~ns}$ is $4 \mathrm{kA}$. which corresponds to $2 \mathrm{kA}$ per wire). The exact current waveforms for the Cornell shots are not known, although the currents' peak values and rise-times correspond to those in our 
simulations. As results in the first chapter show, the shape of the current waveform can play a significant role in determining the dynamics of the wire explosion and plasma deposition. For this reason, even though our analysis differs significantly from Cornell results - generally by a factor of two in the high-current experiment and by a factor of ten in the low-current experiment -, it may still provide a reasonable picture of the process for the parameters in our model. Finally, unlike in the previous two chapters, we are not concerned with the wires being confined to a circular array, so the notion of the separation distance between the wires is not applicable. (In the two-wire "low-current" Cornell experiment, it far exceeds the radius of the wire at the peak current.)

Our results show no significant differences among the gold-plated, pure tungsten and pure gold wires in the high-current experiments; in the lowcurrent experiment, the single-material wires expand faster than the goldplated wire, but maintain approximately the same wire radius. Such quantities as linear density, core expansion rate, and corona mass are very similar for all three cases in the high-current experiment. In the low-current experiment, we were not able to successfully compute linear density and corona mass due to the limitations of our model, in particular due to the bonding model. However, we believe that the overall process and properties, such as the expansion of the wire and the formation of the plasma, predicted by the model are reasonable.

\subsection{High-Current Results}

The linear high-current Shot 1 and the radial expansion of the $5 \%$ gold-plated wires is shown in Figure 3.1. The gold zones initially expand slightly faster than the tungsten zones because they form a skin layer and thus carry most of the current. The tungsten then expands at a constant rate of about 4.2-5.1 $\mathrm{mm} / \mu \mathrm{s}$, reaching the maximum tungsten wire radius of approximately 570 $\mu \mathrm{m}$ covered by $100 \mu \mathrm{m}$ of gold at the current peak. There appears to be no repinch effect for the tungsten, although the gold expands to about 350-450 $\mu \mathrm{m}$ after about $13 \mathrm{~ns}(22 \mathrm{kA}$ ), then contracts slightly, and after about $27 \mathrm{~ns}$, expands linearly with the tungsten.

Figure 3.2, which shows the density-radius contours at different currents, demonstrates that the core steadily expands up to the $60 \mathrm{kA}$ point, after which time. the core is continuously vaporized until all of the tungsten is 


\begin{tabular}{|l|l|l|}
\hline Current (kA) & Linear Density $(\boldsymbol{\mu g} / \mathbf{c m})$ & Corona Mass (\%) \\
\hline 20 & 5.61659 & 8.75 \\
\hline 40 & 11.2009 & 17.50 \\
\hline 60 & 21.2286 & 33.17 \\
\hline 80 & 50.2719 & 78.55 \\
\hline 100 & 61.7059 & 100.00 \\
\hline
\end{tabular}

Table 3.1: Corona linear density and percent mass for "high-current" Shot 1 for $5 \%$ gold-plated wire.

turned into plasma by the time the peak current of $100 \mathrm{kA}$ is reached. The steep "tail" of the contours corresponds to the gold zones which are pushed forward by the expanding tungsten, although they themselves do not expand any further.

As in the previous chapters, we calculated the corona linear density, Figure 3.3, and the percent mass deposited in the corona for the wire, Table 3.1. (The percent mass may not be in absolute agreement with the quantity obtained by dividing the full linear density by the original linear density of $6.37 \times 10^{-5} \mathrm{~g} / \mathrm{cm}$ due to numerical integration error of up to $10 \%$ when using Tecplot CFD Analyzer.) The data demonstrate that most plasma growth occurs between 40-50 and $100 \mathrm{kA}$ where the percent mass of plasma deposited into the corona increases by a factor of five. As mentioned before, the initial slower growth rate is due to the current flowing through the gold coating without penetrating and, hence, heating the tungsten wire.

Although the Cornell waveform is not known, other than the fact that the peak current of $100 \mathrm{kA}$ is reached between 50 and $60 \mathrm{~ns}$, Table 3.2 shows the comparison of the core and full wire (core plus corona) radii at $51.2 \mathrm{~ns}$. We interpret the Cornell results based on a backlighting image: this nakedeye analysis introduces some error into the interpretation. Unfortunately, we do not have any data on linear density or corona mass from the Cornell experiment. Finally, we also have a backlighitng image at $59.2 \mathrm{~ns}$, but the differences between the 51.2 and 59.2 ns images are very minor and we therefore only concentrate on the 51.2 ns image.

Although the core radii are quite comparable, the predicted wire radius is almost twice that actually observed. The reason for this difference, we believe, is that in the experiment, due to tungsten impurities. the current does not penetrate the wire as much as in the model, resulting in less ohmic 


\begin{tabular}{|l|l|l|}
\hline Property & Model & Experiment \\
\hline Core radius $(\mu \mathrm{m})$ & 80.5 & $84-120$ \\
\hline Full wire radius $(\mu \mathrm{m})$ & $500-590$ & $240-300$ \\
\hline
\end{tabular}

Table 3.2: Core and wire length comparison for "high-current" Shot 1 for $5 \%$ gold-plated wire.

heating, which leads to a slower core expansion rate and slower deposition of the tungsten into the corona. In addition, we do not know for sure if in the Cornell experiment, the core has already reached its maximum radius and is being vaporized when the backlighting image is taken at $51.2 \mathrm{~ns}$ or if the core continues to expand at this point. In the former case, i.e. if the maximum core radius is not reached before $51.2 \mathrm{~ns}$, the average expansion rate would be only $1.45-2.62 \mathrm{~mm} / \mu \mathrm{s}$, which is well below the pure-tungsten expansion rates of $4-5 \mathrm{~mm} / \mu$ s predicted by the model and observed in Cornell experiment as described in Chapter 1. Thus we are inclined to believe that the core radius has reaches its maximum before $51.2 \mathrm{~ns}$ and the core is being deposited into the corona without further expansion.

During the expansion of the gold zones, the electron temperature reaches at most $45 \mathrm{eV}$ and then declines to that of the adjacent tungsten zones. The electron temperature in the core stays below $6-10 \mathrm{eV}$ and reaches up to 35 $\mathrm{eV}$ in the corona at all times. The radiation temperature throughout the wire remains within similar limits, reaching a maximum of $35 \mathrm{eV}$ during the expansion of the gold zones, then decreasing to about $8 \mathrm{eV}$. Throughout the expansion of the tungsten wire, it never reaches above its maximum of 23 $\mathrm{eV}$ which occurs at $25 \mathrm{~ns}$. The timing of the sudden sharp rise in electron and radiation temperatures at the onset of the current, Figures 3.4 and 3.5, coincides with the time when the wire begins to expand, Figure 3.1. We believe that this spike is due to the breakdown of the skin laver (and the current beginning to flow through the wire) and the associated decoupling of radiation and electron temperatures. We note that these temperatures are significantly lower than in the impure (carbon-coated) tungsten case. as in Chapter 2.

In addition to $5 \%$ gold-plated tungsten, we modeled pure tungsten and pure gold wires under the same current shots. In summary, we did not observe any significant differences between the three cases, while there are real differences in wire dynamics observed in experiments. We believe that this is 


\begin{tabular}{|l|l|l|l|l|}
\hline Property & $\begin{array}{c}\text { 5\% Au } \\
\text { Expt }\end{array}$ & $\begin{array}{l}\text { 5\% Au } \\
\text { Model }\end{array}$ & $\begin{array}{l}\text { 100\% W } \\
\text { Model }\end{array}$ & $\begin{array}{l}\text { 100\% Au } \\
\text { Model }\end{array}$ \\
\hline Core radius $(\mu \mathrm{m})$ & $84-120$ & 80.5 & 78.1 & 140 \\
\hline Full wire raidus $(\mu \mathrm{m})$ & $240-300$ & $500-590$ & 650 & 625 \\
\hline Core expansion rate $(\mathrm{mm} / \mu \mathrm{s})$ & Unknown & $4.2-5.1$ & $5.2-6.3$ & $6.0-6.4$ \\
\hline
\end{tabular}

Table 3.3: Core radius, wire radius (at $51.2 \mathrm{~ns}$ ), and expansion rate comparison for "high-current" Shot 1 for $5 \%$ gold-plated, pure tungsten, and pure gold wires.

primarily due to the fact that gold and tungsten have very similar material properties when modeled but very different actual properties in the experiments. For instance, we assume that both tungsten and gold are completely pure, while in reality gold tends to be much more pure than tungsten. As observed previously, the purity of the wire affects the distribution of the current flow which in turn affects plasma formation and wire expansion rates. Furthermore, we used the same opacity table for both tungsten and gold, which may have affected our results negatively.

Figures 3.6 and 3.7 show the radial expansion of the wires for pure tungsten and pure gold wires, respectively. There are no apparent differences in the overall expansion process among pure tungsten, pure gold, and goldplated tungsten wires. However, the core expansion rates are approximately $5.2-6.3 \mathrm{~mm} / \mu \mathrm{s}$ for pure tungsten wire and $6.0-6.7 \mathrm{~mm} / \mu \mathrm{s}$ for pure gold wire, both somewhat higher than for gold-plated tungsten wire but also very close to the observed expansion rates in other Cornell experiments. Thus, confirming the result from the previous chapter, our model approximates core expansion rates and the orerall expansion process better for pure, singlemetal wires than for dual-material wires. The results are summarized in Table 3.3.

The linear density profiles are very similar for pure tungsten and pure gold wires and are shown in Figures 3.8 and 3.9. The data for full corona linear density and the percent mass in the corona are shown in Tables 3.4 and 3.5. The corona linear densities differ by at most $5 \mu \mathrm{g} / \mathrm{cm}$ from the pure gold wire; the difference is especially noticeable during the most intense part of the vaporization, between 40 and $80 \mathrm{kA}$. The differences in corona mass are also small; most of the corona growth occurs after the $40 \mathrm{kA}$ current reached, though unlike tungsten wires, the pure gold wire never entirely vaporizes, 


\begin{tabular}{|l|l|l|l|}
\hline Current (kA) & $\mathbf{5 \%} \mathbf{A u}(\mu \mathrm{g} / \mathbf{c m})$ & $\mathbf{1 0 0 \%} \mathbf{W}(\mu \mathrm{g} / \mathbf{c m})$ & $\mathbf{1 0 0 \%} \mathbf{A u}(\mu \mathbf{g} / \mathbf{c m})$ \\
\hline 20 & 5.61659 & 2.124244 & 3.18951 \\
\hline 40 & 11.2009 & 11.9971 & 10.8640 \\
\hline 60 & 21.2286 & 29.3441 & 25.751 \\
\hline 80 & 50.2719 & 50.2687 & 45.6369 \\
\hline 100 & 61.7059 & 61.8889 & 58.8853 \\
\hline
\end{tabular}

Table 3.4: Full corona linear density for "high-current" Shot 1 for $5 \%$ goldplated, pure tungsten, and pure gold wires.

\begin{tabular}{|l|l|l|l|}
\hline Current (kA) & $\mathbf{5 \%}$ Au (\%) & $\mathbf{1 0 0 \% ~ W ~ ( \% ) ~}$ & $\mathbf{1 0 0 \%}$ Au (\%) \\
\hline 20 & 8.75 & 4.75 & 4.99 \\
\hline 40 & 17.50 & 19.82 & 17.05 \\
\hline 60 & 33.17 & 46.10 & 40.44 \\
\hline 80 & 78.55 & 78.99 & 71.69 \\
\hline 100 & 100.00 & 100.00 & 92.46 \\
\hline
\end{tabular}

Table 3.5: Percent mass in the corona for "high-current" Shot 1 for $5 \%$ goldplated, pure tungsten, and pure gold wires.

leaving an $8 \%$-mass core. We do not know what particular property of gold accounts for this effect.

Unfortunately. we do not have any experimental data for this $100 \mathrm{kA}$, $60 \mathrm{~ns}$ shot for pure gold wires. However, we do have Cornell experimental data for a steeper, $40 \mathrm{~ns}$, pulse for $10 \mu \mathrm{m}$ pure tungsten wires. These data, taken near the peak current show that a dense core, about $30-40 \mu \mathrm{m}$ (half of our core radius at $51.2 \mathrm{~ns}$ ) in radius and containing $50 \%$ of the wire mass, is present; the linear density profile is about a factor of two smaller than the one shown in Figure 3.8. While these results do differ somewhat from our model, the current waveform probably accounts for most the differences since the extra $10-20 \mathrm{~ns}$ of heating at high currents $(80-100 \mathrm{kA})$ present in our model can easily melt the remaining $30 \mu \mathrm{m}$ core and deposit the material into the corona.

The electron and radiation temperature profiles for the pure tungsten and pure gold wire resemble those for the gold-plated wire as seen in Figures 3.4 and 3.5. As the current is turned out, there is a spike in electron temperature in the outer zones. reaching $45-50 \mathrm{eV}$. Then, there is a decline, with core temperatures staring below $8-10 \mathrm{eV}$ and corona temperatures staying below 
$30-35 \mathrm{eV}$. The radiation temperature also has an initial spike to $30 \mathrm{eV}$, but then stays below its maximum of $28 \mathrm{eV}$ for pure tungsten and $23 \mathrm{eV}$ for pure gold. As in the gold-plated case, the initial spike in electron and radiation temperatures is probably due to the decoupling of the radiation from the electrons as the skin layer is broken and the current begins flowing through the wire.

It would be interesting to model gold-plated tungsten wires where gold comprises $10 \%, 20 \%, 30 \%$, etc. of the wire and to see if there are any trends in the explosion dynamics. Our initial results suggest that there should be no significant differences since the properties of gold-plated, pure tungsten and pure gold wire explosions are very similar; however, with improved conductivity models and the correct gold opacity data, the model may predict different, more correct results. We do not know of experimental data for these wires with higher gold composition.

\subsection{Low-Current Results}

In this section we describe our model results for the behavior of $10.247 \mu \mathrm{m}$ $5 \%$ gold-plated, pure tungsten and pure gold wires under the "low-current" Shot 2, which consists of a linear current rising to $2 \mathrm{kA}$ in $267.6 \mathrm{~ns}$. This peak exactly corresponds to the peak in the Cornell experiment, although as in the previous section, the Cornell waveform is not known exactly.

Figure 3.10 shows the expansion of the $5 \%$ gold-plated wire under Shot 2. As the figure demonstrates, for the first $40 \mathrm{~ns}$, the current is forming a skin layer, not penetrating far into the wire. After the 40 ns point, however, wire expansion begins, and unlike in the high-current experiment, Figure 3.1, there is no repinching of the gold zones before the expansion of the tungsten zones. At the peak current, the gold region extends to the radius of 0.60 $1.54 \mathrm{~cm}$, while the tungsten reaches only $500 \mu \mathrm{m}$. The uncertainty in the radius of the corona comes from the fact that the last few gold zones have extremely low densities, below $10^{-8} \mathrm{~g} / \mathrm{cm}^{3}$, which suggest that in reality the gold is being blown away from the wire and cannot be considered part of the wire plasma, Figure 3.11. This figure shows density as a function of the radius at the peak current of $2 \mathrm{kA}$ In addition, it demonstrates the peculiar feature of the bonding model: the density in the tungsten zones alternates between being on the order of $10^{-3} \mathrm{~g} / \mathrm{cm}^{3}$ and $10^{1} \mathrm{~g} / \mathrm{cm}^{3}$, i.e., near solid density. In order to keep the material from blowing apart, the bonding model introduces 
this special feature which forces the density to alternate between the zones, suggesting that some solid material is present. The actual density is on the order of $10^{-3} \mathrm{~g} / \mathrm{cm}^{3}$, and the line shows the correct density as a function of the radius. The square points correspond to the tungsten zones, which, as mentioned above, extend to about $500 \mu \mathrm{m}$, while the circles correspond to the gold zones, which seem to reach beyond $1 \mathrm{~cm}$.

This "alternating density" feature of our model prevents us from accurately calculating the linear density in the tungsten zones because the Tecplot CFD Analyzer produces results which are far from the actual total linear density of $6.37 \times 10^{-5} \mathrm{~g} / \mathrm{cm}$ for the entire wire; for instance, if we keep the high-density zones, the total linear density given by Tecplot is $6.20 \times 10^{-2}$ $\mathrm{g} / \mathrm{cm}$, while if we remove the high-density zones, the total linear density falls to $1.464 \times 10^{-5} \mathrm{~g} / \mathrm{cm}$. For this reason, it is impossible to accurately calculate the corona linear density and percent mass of the wire in the corona. Our attempts to modify various parameters in our model (such as the number of zones, the feathering factor, the minimum density per zone, etc.), have not produced results that get rid off this effect of the bonding model. Therefore, we believe future modifications of the code will be necessary to produce accurate density plots for this current shot.

Despite this inadequacy in our model, we can estimate the boundary between the wire core and the corona. From Figure 3.11, we estimate that the core radius is $140 \mu \mathrm{m}$. Using the backlighting image at $267.6 \mathrm{~ns}$ and 2 $\mathrm{kA}$ from the Cornell experiment, we estimated the core and wire radius in the experiments: this comparison is shown in Table 3.6. Both core and wire radii in the model are about ten times greater than experimental measurements; there are at least two possible explanations for this observation. The first is again the presence of impurities in the tungsten region in the actual wire; these impurities probably carry most of the current once the current penetrates the gold coating and prohibit the tungsten from heating and expanding rapidly during all of the time in Shot 2 . This conclusion is justified as follows: the current throughout Shot 2 is very small - below $2 \mathrm{kA}$ - and, as discussed in Chapter 2, the model predicts that the current penetrates the tungsten (which is covered by an impurities layer) only above $20 \mathrm{kA}$ Furthermore, the wire in the model expands for a very long time - more than 250 ns - which can lead to the great discrepancy between model and experiment. Thus, we believe our results are consistent with the weaknesses of the model and the actual experimental set-up. Another explanation for these effects may be due to TRAC II bonding, conductivity and Equation of State (EOS) 


\begin{tabular}{|l|l|l|}
\hline Property & Model & Experiment \\
\hline Core radius $(\mu \mathrm{m})$ & 140 & $10-20$ \\
\hline Full wire radius $(\mu \mathrm{m})$ & $5000-15000$ & $70-75$ \\
\hline
\end{tabular}

Table 3.6: Core radius and wire radius comparison for "low-current" Shot 2 for $5 \%$ gold-plated wire.

models at such low currents. It may simply be the case that our model is not applicable to cases where the current remains very small (below $5 \mathrm{kA}$ ) for a very long time (more than $100 \mathrm{~ns}$ ).

The electron and radiation temperatures for $5 \%$ gold-plated wire for Shot 2 are shown in Figures 3.12 and 3.13. They demonstrate that both temperatures remain at $20-30 \mathrm{eV}$ below those in "high-current" Shot 1 . The peak electron temperature of $15 \mathrm{eV}$ and the peak radiation temperature of $10 \mathrm{eV}$ occur right before the wire begins to expand, at about $40 \mathrm{~ns}$. As in the "highcurrent" shot in the previous section, this spike is due to the disintegration of the skin layer and the decoupling of electron and radiation temperatures Throughout the expansion, the electron temperature stays below $12 \mathrm{eV}$, and radiation temperature below $1 \mathrm{eV}$. Such low temperatures suggests that the expansion of the wire is very slow, and in fact, on the basis of Figure 3.11 and similar graphs for $0.5,1.0$, and $1.5 \mathrm{kA}$, we estimate the average core expansion rate to be about $0.55 \mathrm{~mm} / \mu \mathrm{s}$, or about ten times slower than in the "high-current" Shot 1. There are two interesting features in the electron and radiation temperature plots; the first is the apparent, relatively small oscillations of the temperature after the expansion has begun and the second is the sudden drop-off in temperature for a ferw of the gold zones. The first is probably related to the bonding model and the resulting variation in density, while for the second we have no clear explanation. Finally, it is interesting to note that the radiation and electron temperatures do not increase as the current grows; this is probably due to the fact that the current remains very small throughout the entire shot.

In the case of pure tungsten and pure gold wires, we obtain results that differ from those of the $5 \%$ gold-plated wire. While the radial expansions of these wires, Figures 3.14 and 3.15 , resemble the expansion of the goldplated wire, Figure 3.10, the composition - the core-corona structure - is quite different. The density contours for the pure tungsten and pure gold wires at the peak current of $2 \mathrm{kA}$ (267.6 ns) are shown in Figures 3.16 and 3.17. First as in the pure-tungsten case in Chapter 1, Figure 1.4, there is 
a small kink at the core-corona boundary which is observed in experiments. Second, the figures definitively show that the core extends to a radius of about $600 \mu \mathrm{m}$, fives times greater than for the $5 \%$ gold-plated wire, and the corona reaches beyond $1 \mathrm{~cm}$ as in the gold-plated case. The density beyond $5 \mathrm{~mm}$ is very small, below $10^{-8} \mathrm{~g} / \mathrm{cm}^{3}$, and, therefore, we may assume the wire ends at about this point. A good comparison of the contours is also shown in Figure 3.18. The large core radius also results in a larger core expansion rate; based on Figures 3.16 and 3.17 and similar graphs for $0.5,1.0$. and $1.5 \mathrm{kA}$, we estimate the expansion rate of these wires to be approximately $2.7-2.8 \mathrm{~mm} / \mu \mathrm{s}$, again about five times faster than for the gold-plated wire. Unfortunately, we do not have a good explanation for this variation in core radius between the single-material and two-material wires, although the electron temperature differences between the two cases may help explain this observation.

Although we do not show the graphs of electron and radiation temperatures for the pure wires, their shapes resemble those for the gold-plated wire, Figures 3.16 and 3.17 below. For the pure tungsten wire, the electron temperature increases as the current intensifies and reaches its maximum of $9.5 \mathrm{eV}$ at about $45 \mathrm{~ns}$, just before the current's penetration and expansion of the wire begins. After that, the temperature declines and remains below the outer zone maximum of $4.2 \mathrm{eV}$ as the wire expands. The radiation temperature follows the same pattern, reaching its maximum of $5.8 \mathrm{eV}$ before declining and remaining steady below $1.2 \mathrm{eV}$ in the outer zones. For pure gold wires, the temperatures follow the same patterns with a peak electron temperature of $10.2 \mathrm{eV}$ and peak radiation temperature of $5.0 \mathrm{eV}$ just before the breakdown of the skin layer, followed by steady temperatures below $1 \mathrm{eV}$. Both peak values for the radiation and electron temperatures are about $5 \mathrm{eV}$ below the peaks for the gold-plated wire, and the steady electron temperature is $5-7 \mathrm{eV}$ below that for the gold-plated wire. These lower temperatures suggest that the plasma is not being produced as quickly as in the goldplated wire, and therefore, the core has more time to expand before being turned into plasma. However, the factor of five difference is rather large, and this explanation may not be satisfactory. The electron and radiation temperatures for the pure wires also exhibit the same sort of oscillations and sudden drop-off as the temperature for gold-plated wire, Figures 3.12 and 3.13. Again, unfortunately, we do not have a good explanation for this effect other than it being a consequence of the EOS and bonding models..

$\mathrm{As}$ is the case with gold-plated wires, the bonding model requires some 


\begin{tabular}{|l|l|l|l|l|}
\hline Property & $\begin{array}{l}\text { 5\% Au } \\
\text { Expt }\end{array}$ & $\begin{array}{l}\text { 5\% Au } \\
\text { Model }\end{array}$ & $\begin{array}{l}\mathbf{1 0 0 \%} \text { W } \\
\text { Model }\end{array}$ & $\begin{array}{l}\mathbf{1 0 0 \%} \text { Au } \\
\text { Model }\end{array}$ \\
\hline Core radius $(\mu \mathrm{m})$ & $10-20$ & 140 & 610 & 570 \\
\hline Full wire raidus $(\mu \mathrm{m})$ & $70-75$ & $5000-15000$ & $4500-10000$ & $4000-10000$ \\
\hline Core expansion rate $(\mathrm{mm} / \mu \mathrm{s})$ & Unknown & 0.55 & 2.80 & 2.74 \\
\hline
\end{tabular}

Table 3.7: Core radius and wire radius comparison for "low-current" Shot 2 for $5 \%$ gold-plated wire.

zones to have higher density than others (by several orders of magnitude), Figures 3.16 and 3.17, and this prevents us from calculating linear density and corona mass accurately. We summarize our results in Table 3.7.

In conclusion, we believe that there are several improvements that are necessary in order for TRAC II to more accurately model the wire explosions discussed in this chapter: better accounting for impurities in the tungsten wires, more accurate conductivity and EOS models at low currents, and improved bonding model that does not force "alternating" densities between the zones. 


\subsection{Figures for Chapter 3}

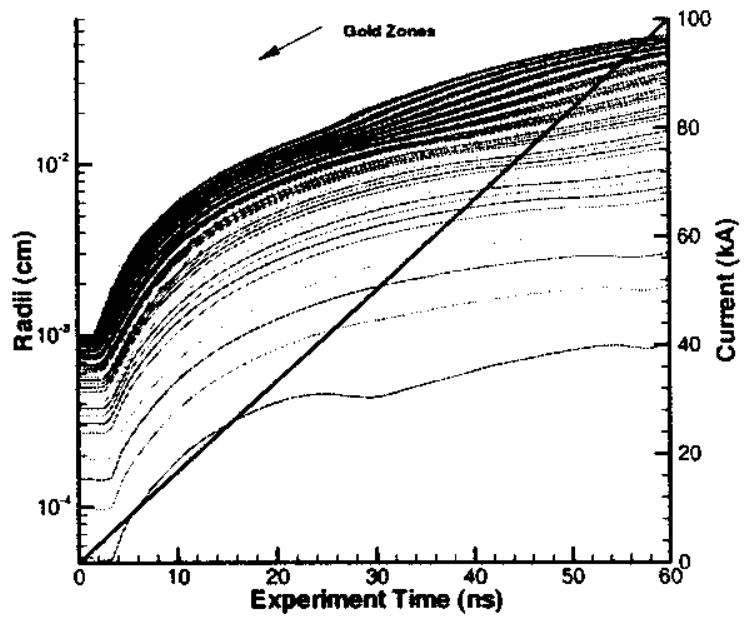

Figure 3.1: Radii (mesh points) as a function of time for "high-current" Shot 1 for $5 \%$ gold-plated wire. 


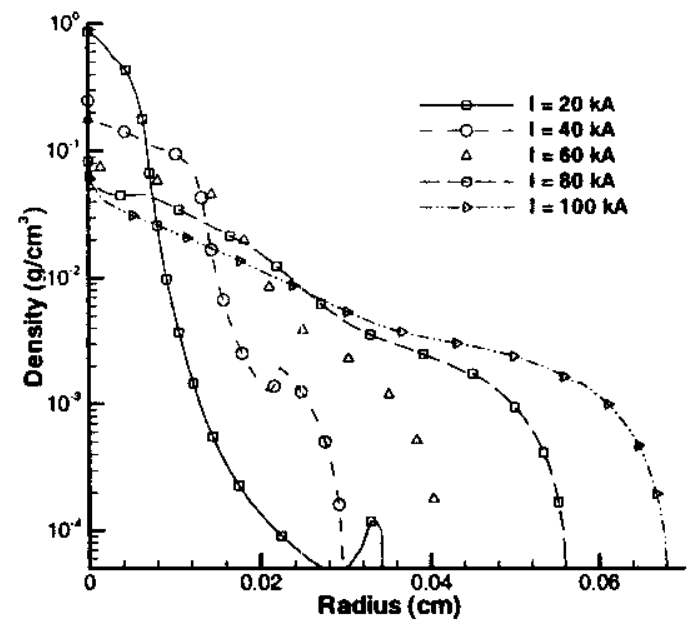

Figure 3.2: Density as a function of the radius for "high-current" Shot 1 for $5 \%$ gold-plated wire at $20,40,60,80$, and $100 \mathrm{kA}$.

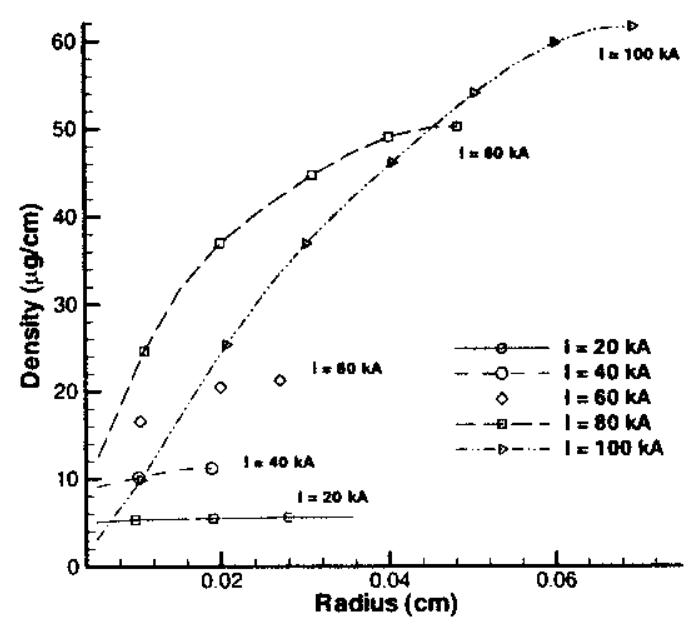

Figure 3.3: Corona linear density as a function of the radius for "highcurrent" Shot 1 for $5 \%$ gold-plated wires at $20,40,60,80$. and $100 \mathrm{kA}$. 


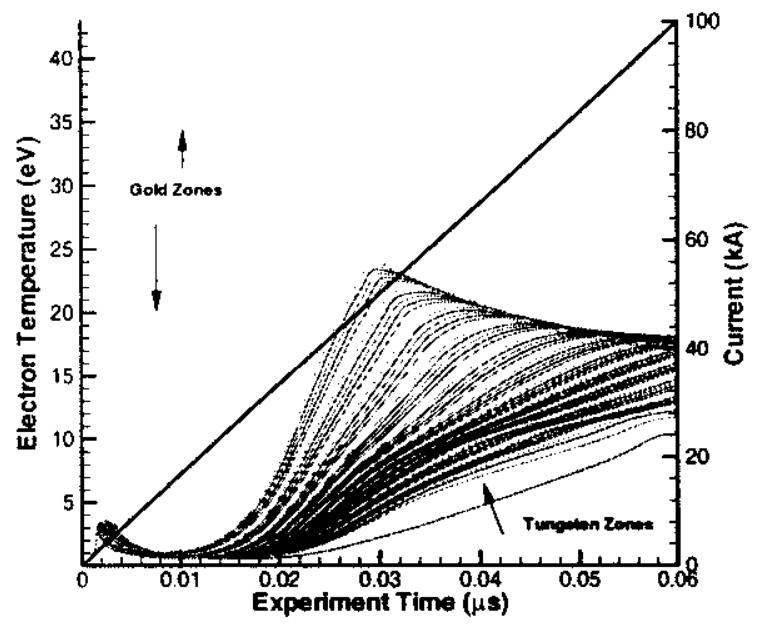

Figure 3.4: Electron temperatures as a function of time for "high-current" Shot 1 for $5 \%$ gold-plated wire.

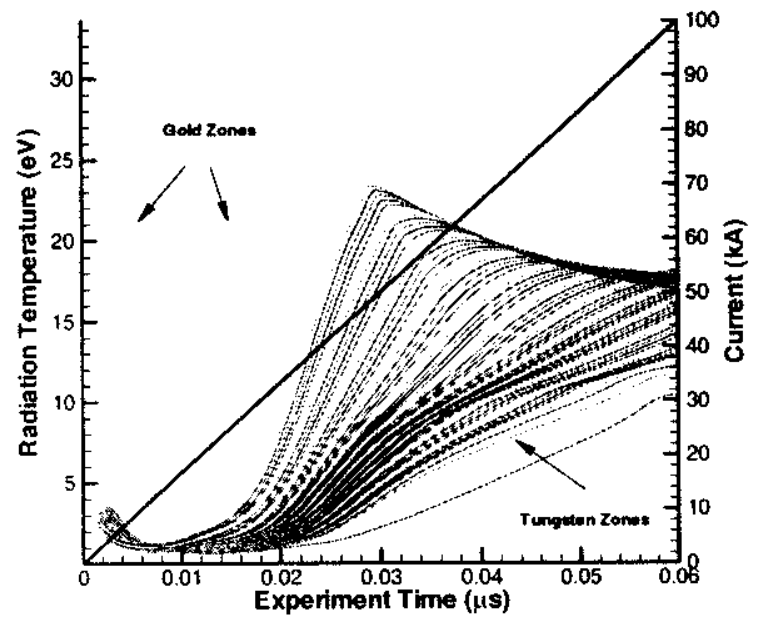

Figure 3.5: Radiation temperature as functions of time for "high-current" Shot 1 for $5 \%$ gold-plated wire. 


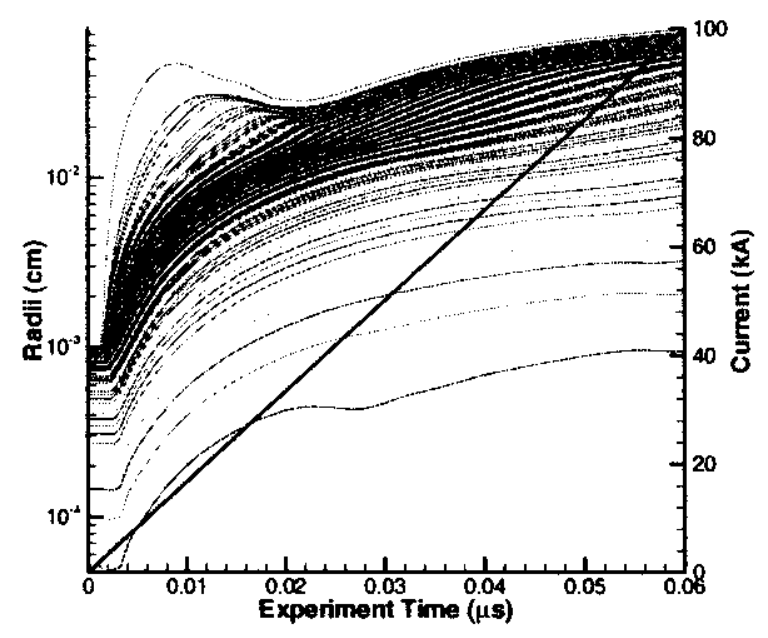

Figure 3.6: Radii (mesh points) as a function of time for "high-current" Shot 1 for pure tungsten wire.

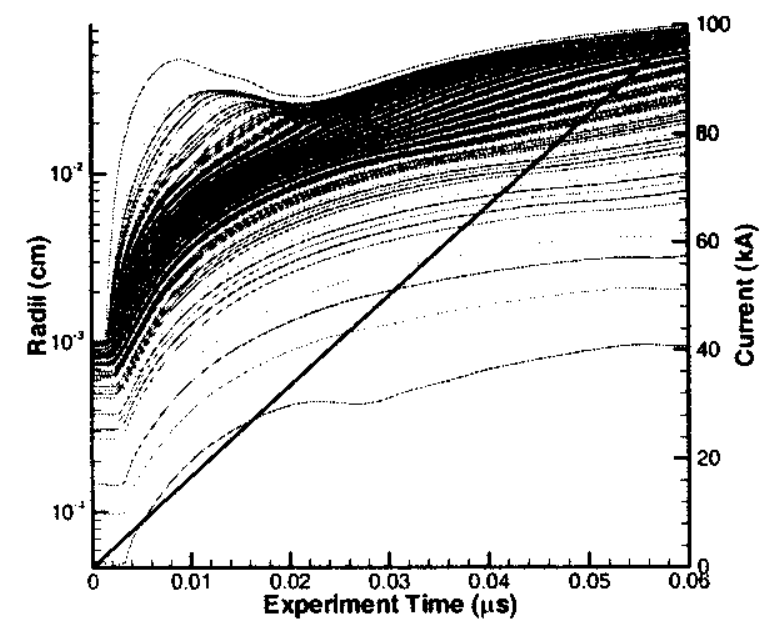

Figure 3.7: Radii (mesh points) as a function of time for "high-current" Shot 1 for pure gold wire. 


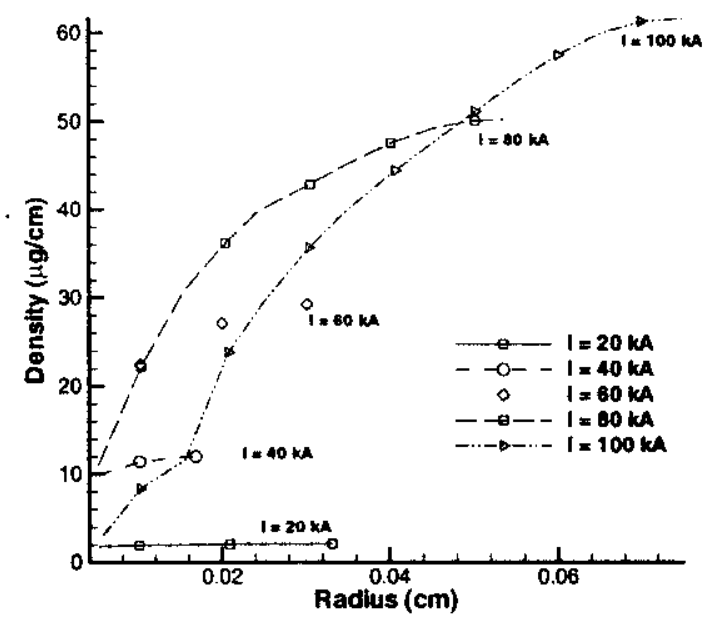

Figure 3.8: Corona linear density as a function of the radius for "highcurrent" Shot 1 for pure tungsten wire at 20,40,60, 80, and $100 \mathrm{kA}$.

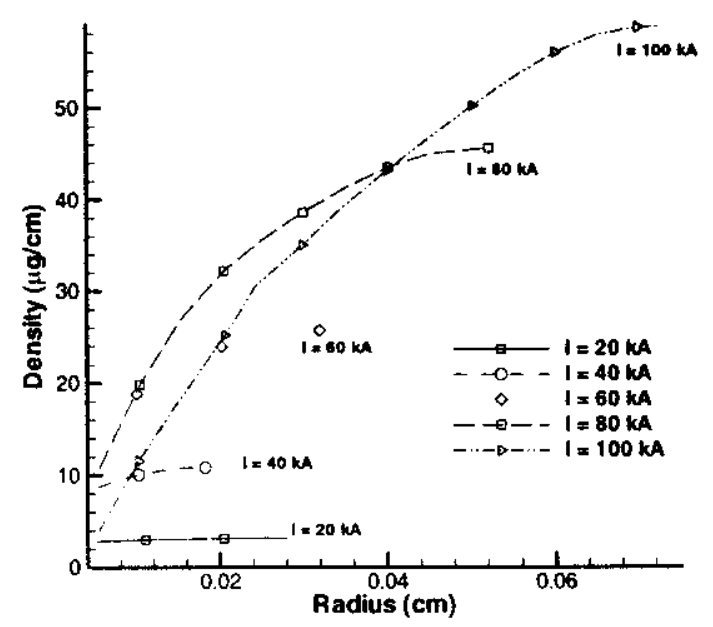

Figure 3.9: Corona linear density as a function of the radius for "highcurrent" Shot 1 for pure gold wire at 20,40,60, 80, and $100 \mathrm{kA}$. 


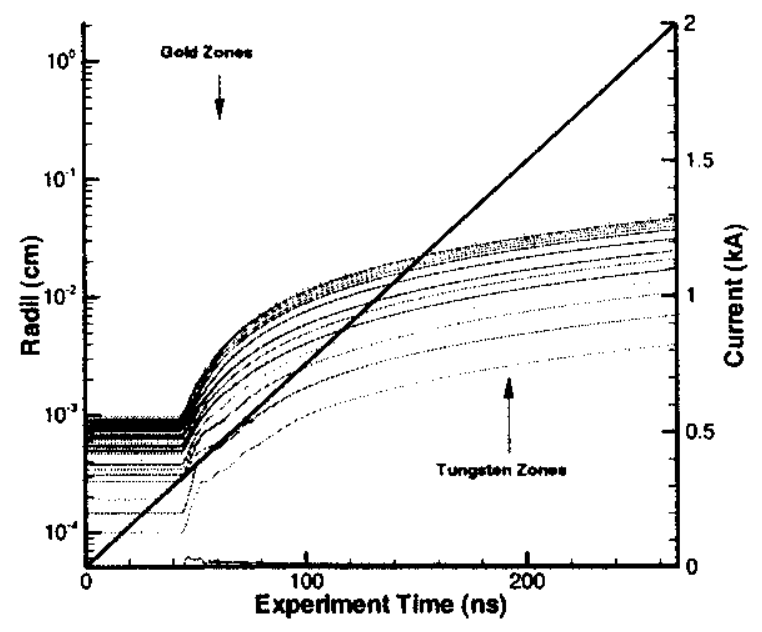

Figure 3.10: Radii (mesh points) as a function of time for "low-current" Shot 2 for $5 \%$ gold-plated wire.

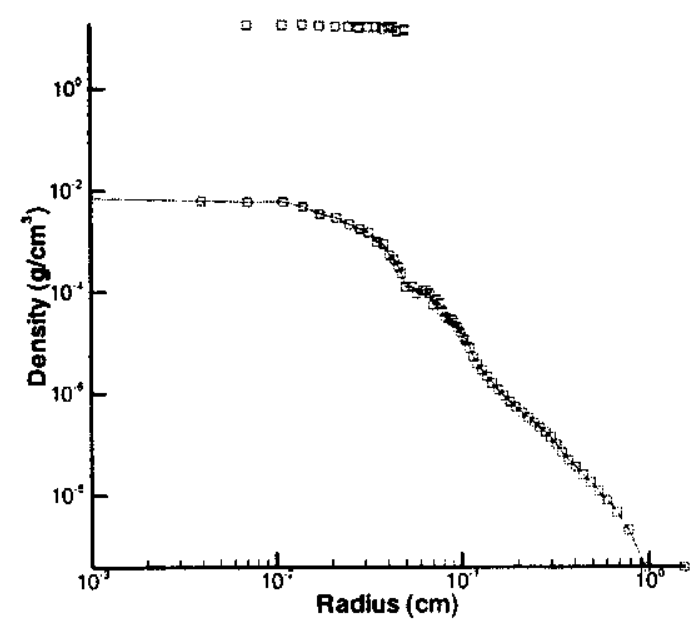

Figure 3.11: Density as a function of the radius for for "low-current" Shot 2 for $5 \%$ gold-plated wire at the peak current of $2 \mathrm{kA}$. 


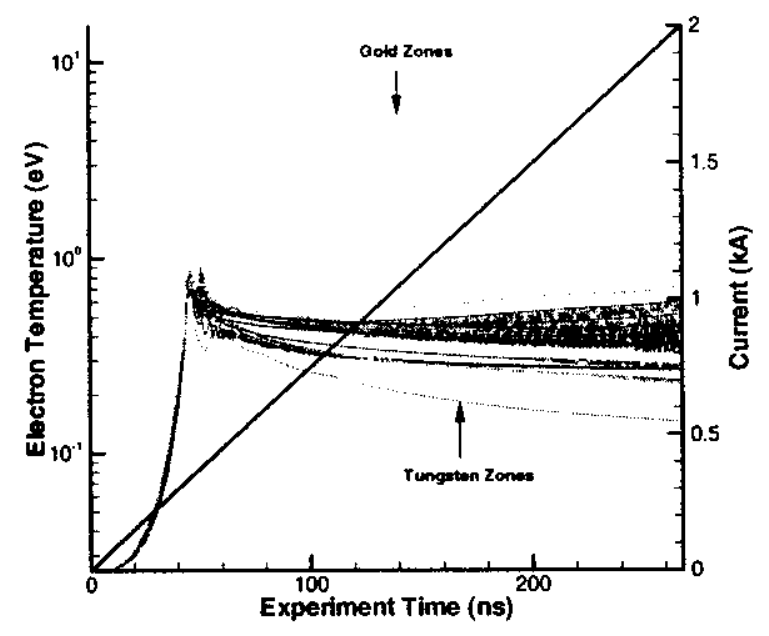

Figure 3.12: Electron temperatures as a function of time for "low-current" Shot 2 for $5 \%$ gold-plated wire.

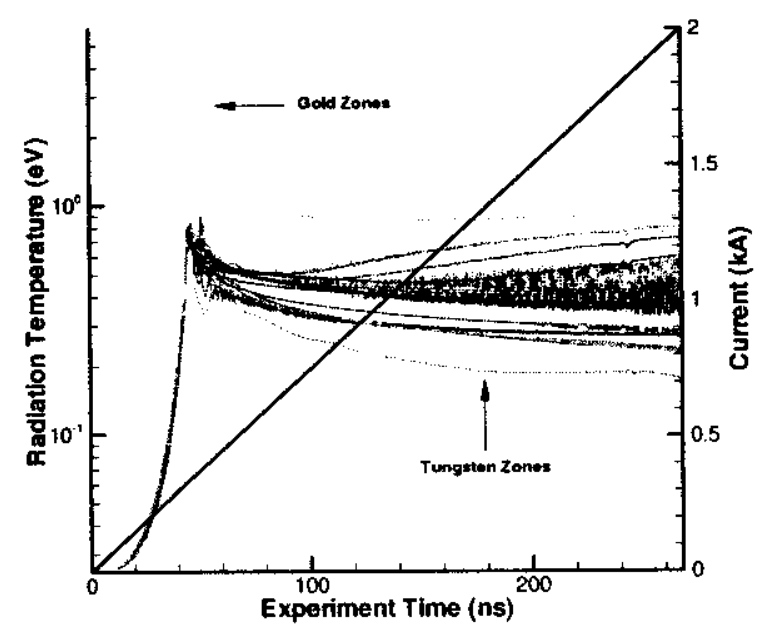

Figure 3.13: Radiation temperatures as a function of time for "low-current" Shot 2 for $5 \%$ gold-plated tungsten wire. 


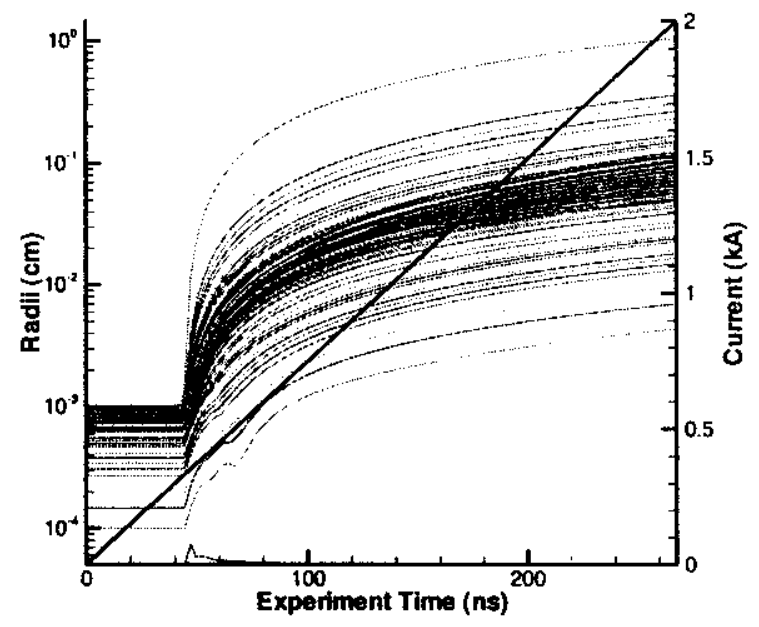

Figure 3.14: Radii (mesh points) as a function of time for "low-current" Shot 2 for pure tungsten wire.

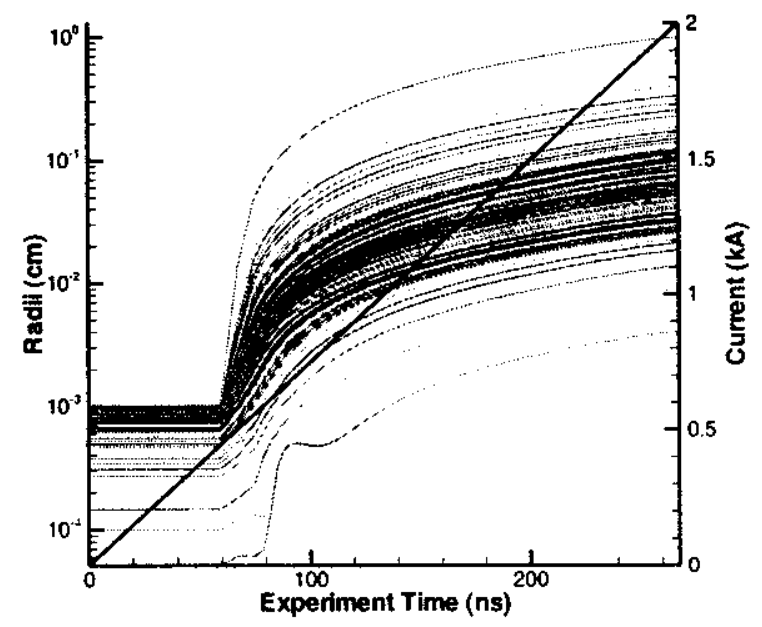

Figure 3.15: Radii (mesh points) as a function of time for "low-current" Shot 2 for pure tungsten wire. 


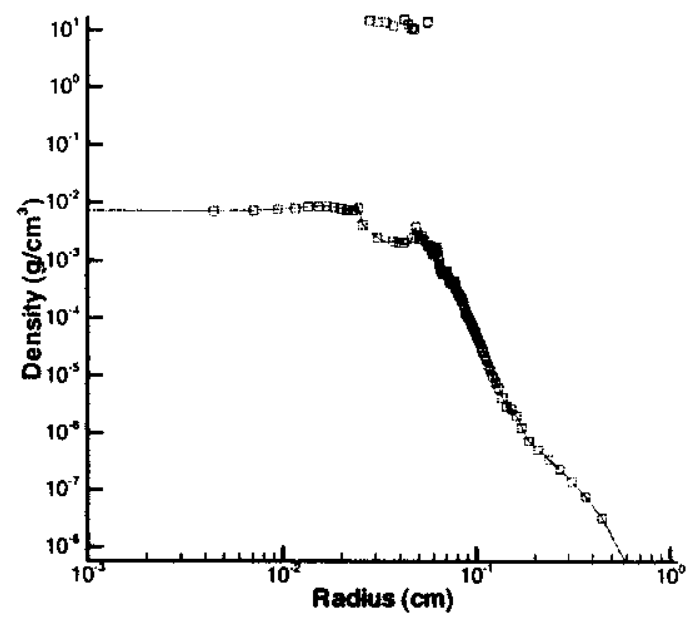

Figure 3.16: Density as a function of the radius for "low-current" Shot 2 for pure tungsten wire.

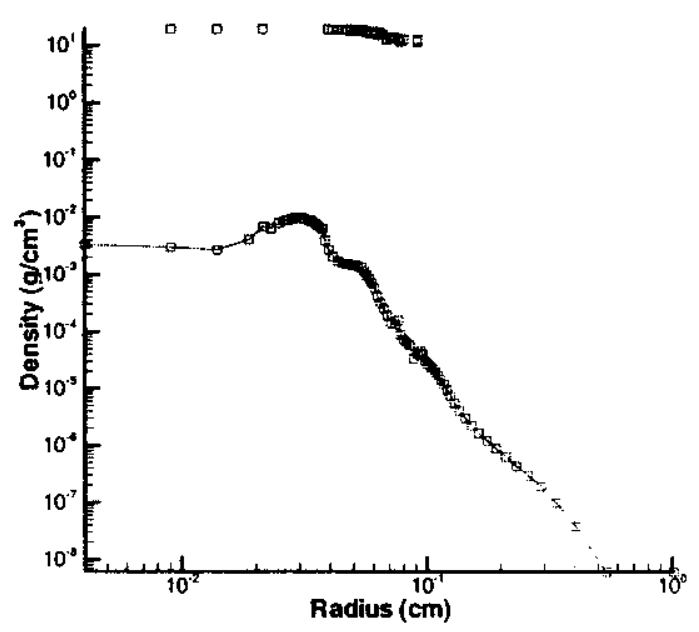

Figure 3.17: Density as a function of the radius for "low-current" Shot 2 for pure gold wire. 


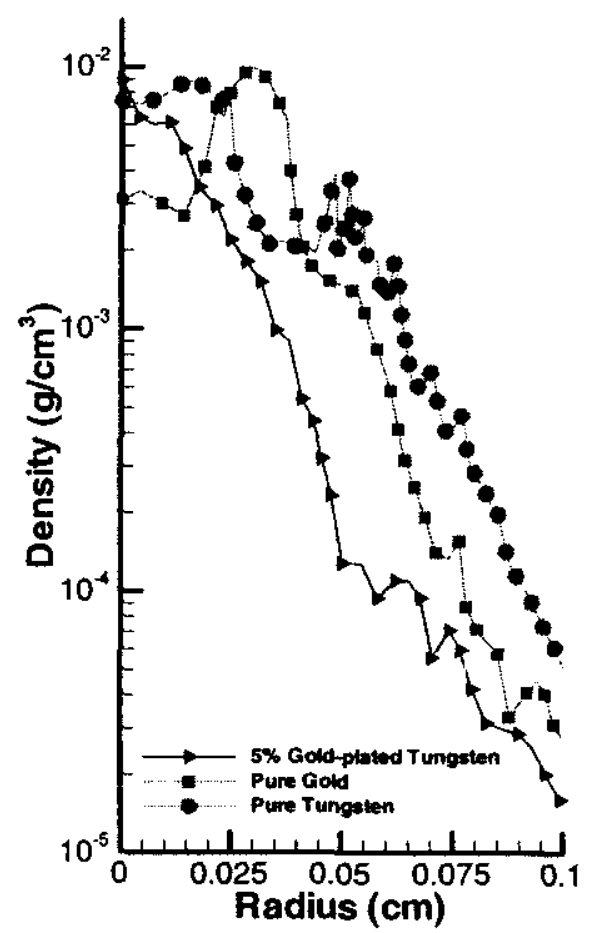

Figure 3.18: Density as a function of the radius for "low-current" Shot 2 for $5 \%$ gold-plated tungsten, pure tungsten, and pure gold wires. 


\section{References}

1. D.D. Ryutov, M.S. Derzon, and M.K. Matzen. "The Physics of Fast ZPinches." Sandia Report SAN98-1632. Albuquerque, NM: Sandia National Laboratories. July, 1998.

2. S.A. Pikuz et al. "Calibrated Density Measurements in Exploding Wire-Initiated Plasinas Using Tungsten Wires." Cornell University Laboratory of Plasma Physics Report LPW 98-03. Ithaca, NY: Cornell University. January, 1999.

3. S.A. Pikuz et al. Other materials based on Cornell University Laboratory of Plasma Physics experiments.

4. M.E. Cuneo et al. Materials based on Sandia National Laboratory experiments. 